\title{
Interactions between Multiple Enrichments in Extended Finite Element Analysis of Short Fiber Reinforced Composites
}

\author{
Matthew G. Pike*, Mason A. Hickman’, and Caglar Oskay \\ Department of Civil and Environmental Engineering \\ Vanderbilt University \\ Nashville, TN 37235
}

\begin{abstract}
This manuscript presents an extended finite element method (XFEM) approach to capture the interactions between fibers in short fiber reinforced composites. Short fiber inclusions are incorporated into the XFEM framework as deformable elastic zero measure objects. Two separate enrichment functions are employed to account for both the presence of fibers within the composite domain and to idealize the progressive debonding along fiber matrix interfaces. This study investigates the accuracy characteristics of the formulation when multiple fiber enrichments and interface debonding enrichments lie within a single element. Accurately capturing multiple enrichments in a single element is particularly important for modeling the failure process of fiber reinforced composites with a significant amount of discontinuous fibers with high aspect ratios. The performance of the proposed XFEM model is assessed by comparing model predictions to the direct finite element method for various interacting fiber configurations. The numerical verification studies indicated that the proposed model displays high accuracy and captures the debonding interactions at fiber-matrix interfaces.
\end{abstract}

Keywords: Extended finite element, interface debonding, discontinuous fiber reinforced composites, short fiber composites, multiple enrichments.

\footnotetext{
*Author address: VU Station B\#351831, 2301 Vanderbilt Place, Nashville, TN 37235. Email: matthew.g.pike@vanderbilt.edu

${ }^{\dagger}$ Author address: VU Station B\#351831, 2301 Vanderbilt Place, Nashville, TN 37235. Email: mason.a.hickman@vanderbilt.edu

${ }^{\ddagger}$ Corresponding author address: VU Station B\#351831, 2301 Vanderbilt Place, Nashville, TN 37235. Email: caglar.oskay@vanderbilt.edu
} 


\section{Introduction}

This manuscript presents an extended finite element method (XFEM) approach to capture the interactions between fibers in short fiber reinforced composites. The interactions due to fiber inclusion and fiber-matrix debonding enrichments of multiple fibers are investigated. The proposed approach enables problems with significant concentration of fiber enrichment by placing multiple fibers within the same element. This approach improves the computational tractability of the XFEM framework compared to implementations in which an element is restricted to contain enrichments from a single fiber.

Short fiber composite materials are frequently used in practice due to favorable mechanical properties, including elastic modulus, load carrying capacity, flexural strength and flexural toughness (see e.g., [1,2] for examples for cementitious materials). Besides the superior mechanical properties, short fiber reinforcement introduces functional properties ranging from crack control, electromagnetic field shielding and self sensing (e.g. [3, 4, 5, 6, 7]).

Micromechanical modeling based on Eshelby's solution of ellipsoidal inclusions combined with the Mori-Tanaka scheme [8, 9, 10], Hashin-Strichman bounds [11] and the use of representative volume elements (RVEs) $[12,13,14]$ are typically used to model the material properties of short fiber reinforced composites. While the micromechanics based effective medium approaches have proven successful, modeling progressive failure along interfaces is challenging. On the other hand, numerical analysis of RVEs using direct discretization of the microstructure is useful for analysis of microstructures with dilute concentrations of inclusions $[15,16,17,18,19]$, but is computationally not feasible in the presence of many, high aspect ratio fibers. The domain discretization must utilize small elements to accurately resolve the fiber response, in addition to ensuring mesh compatibility between the fibers and the matrix.

An alternative approach is applying the XFEM principles to model the behavior of fiberreinforced composites. XFEM provides an approximation basis enrichment strategy that eliminates the need to discretize the individual fibers and ensures mesh compatibility between the fibers and matrix phase. The XFEM approach enriches the standard finite element basis with nodal enrichment functions capable of representing inhomogeneities and discontinuities within the problem domain without explicitly representing them through meshing [20, 21, 22]. To retain the local character of the base finite element formulation, the partition of unity principle [23] is employed. With the partition of unity principle, the original form of the enrichment function, which is known a-priori to represent the local behavior accurately, is recovered. XFEM has been widely employed to model strong (e.g., cracks) and weak discontinuities (e.g., inclusions) [24, 25, 26, 27, 28].

The performance of short fiber reinforced composites are significantly affected by interface 
properties. Cohesive zone modeling has been the traditional approach to idealize progressive debonding along inclusion interfaces. Zero-thickness elements between solid elements that discretize the neighboring domains describe the separation between two surfaces and relate surface tractions to the displacement jumps through a softening constitutive equation (i.e., a cohesive law). Various cohesive laws for cohesive zone modeling of fiber reinforced composites are discussed in $[29,30,31]$, among others. Methods to model cohesive behavior using XFEM have been previously utilized for crack growth (see e.g. [32, 33]). XFEM and cohesive zone modeling has also been introduced by $\mathrm{Zi}$ and Belytschko [34] for a formulation of crack tip elements for cohesive cracks and for partially cracked XFEM elements with cohesive cracks by Asferg et al. [35].

Capturing the mechanical behavior of high density, high aspect ratio fiber reinforced composites in a computationally efficient way requires that the underlying XFEM formulation accommodates the presence of many fibers that are close to each other. A way to alleviate numerical problems that arise from the presence of multiple enrichment functions within the same finite element is local mesh refinement. Within the XFEM framework, the discretization around enrichments are made fine enough that multiple enrichments do not occur within the same element [36]. Other XFEM approaches have been proposed to capture the effect of multiple cracks in the same element, including intersecting cracks and crack growth $[37,38]$. The integration of elements with multiple cracks is achieved by splitting up the element domain into sections and using higher order integration [39, 40]. Hiriyur et al. [41], proposed a method to account for multiple inclusions in the same element domain by introducing additional degrees of freedom for each inclusion enrichment, removing the need to finely discretize the domain around neighboring inclusions.

Embedment methods for fibers in reinforced composites have also been proposed to eliminate the need to discretize individual fibers. Two and three dimensional models have been developed for embedded fibers that include the modeling of fiber slip in the domain by adding additional degrees of freedom [42, 43, 44, 45]. Fiber composite modeling using the partition of unity method was proposed by Radtke et al. [46, 47], where high aspect ratio fibers were modeled as zero measure elastic inclusions for idealizing fiber reinforced composite behavior, eliminating the need to discretize individual fibers. To account for the strong discontinuity present due to tangential debonding at the fiber-matrix interface a Heaviside enrichment function was used. Tangential slip was modeled with a non-linear cohesive law while the normal fiber-matrix interface separation was suppressed. The present manuscript improves on this approach, by considering progressive interfacial separation in both normal and tangential directions. Similar in principles to the XFEM modeling, other methods to embed discontinuities have been proposed (e.g., [48, 49, 50]). Fish and coworkers proposed a mathematical homogenization based approach to include weak discontinuities in a heterogeneous domain [51], 
and employed the s-version finite element method to embed strong discontinuities in a finite element mesh [52].

Pike and Oskay [53] proposed an XFEM model for rigid short fibers in an elastic domain for random fiber composite materials. A progressive failure model for random short fiber reinforced composite materials for elastic deformable fiber inclusions has been previously proposed in Ref. [54]. This investigation restricted element enrichments to a single fiber (including both fiber and debonding enrichments) but did not study the effects of enriching elements to account for multiple closely positioned and interacting fibers.

In this manuscript, we present the formulation and implementation of an XFEM based enrichment coupling model to capture the interactions between short fibers in composites. In a 2-D setting, elements in the domain are permitted to contain multiple fiber inclusions, where the inclusions are modeled as elastic objects of zero measure. Inclusion and debonding enrichment functions are introduced to model the elastic fiber inclusions and the progressive normal and tangential debonding of the fiber in the matrix. Using this process, the debonding relationship is modeled using cohesive laws. Numerical integration procedures are provided for accurate evaluation of the system response for randomly positioned fibers, including multiple fibers that occupy the same element. Fiber configurations where multiple fibers occupy the same element are numerically investigated using the proposed XFEM model and are assessed against the direct finite element method. With the ability to account for multiple zero measure inclusions within the same element in the domain, the fiber volume fraction percentage in a RVE can be increased without a proportional increase in number of elements in the XFEM domain. The key features of the current formulation therefore include: (1) Presence of multiple zero measure inclusions within the same element in XFEM; and (2) Enrichment functions that account for traction-separation behavior and the strain discontinuity at fiber-matrix interfaces.

The remainder of this manuscript is organized as follows. In Section 2, the XFEM method is discussed, and the enrichment functions employed to model the presence of the inclusions and the progressive debonding process are introduced. Section 3 provides the governing equations and model formulation. The computational formulation is discussed in Section 4, including the formulation of fiber deformation and cohesive tractions, numerical integration and the treatment of partially enriched elements. Numerical verification studies to assess the performance of the proposed approach and enrichment interactions are presented in Section 5. Conclusions and future research directions in this area are discussed in Section 6.

\section{XFEM for Multiple Fibers in an Element}

Modeling short fiber reinforced composites with very high aspect ratios (Fig. 1a) through direct resolution of the fiber geometry using the finite element method is impracticable, particularly 


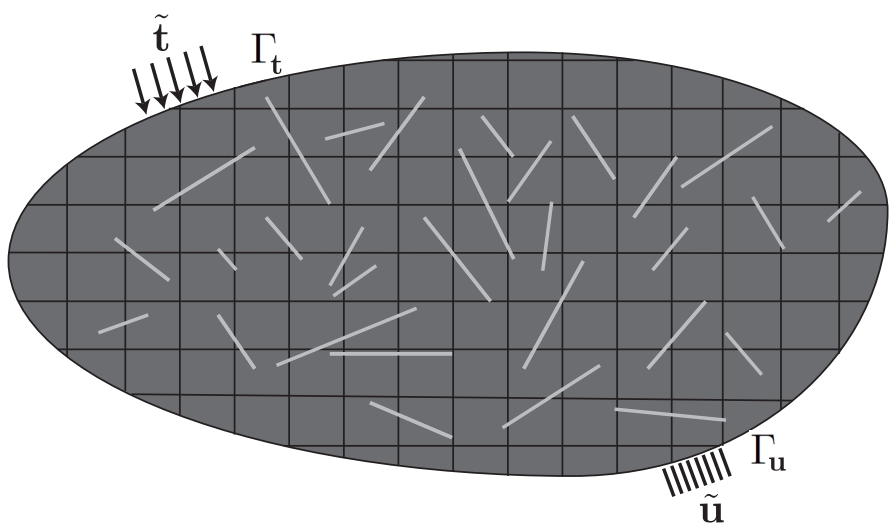

(a)

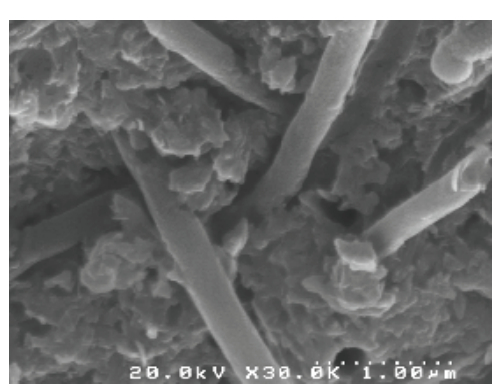

(b)

Figure 1: (a) Domain and XFEM discretization of the short fiber reinforced composite medium; and (b) short fiber reinforced cement composite [55].

when a large number of fibers is present. In this manuscript, we employ XFEM to eliminate the need to conform the discretization to the individual fibers. The XFEM is employed to describe the presence of the fiber inclusions and to idealize the fiber-matrix debonding process.

In an XFEM domain with random short fibers (Fig. 1a), the fibers often lie in the same elements in a uniform grid domain. The random dispersion of numerous fibers is typical for a short fiber reinforced composite (Fig. 1b). A domain with multiple fibers in elements can be eliminated by sufficiently refining the mesh to ensure no two fibers are in the same element. To avoid remeshing of the domain, we seek to account for the interaction behavior of multiple fibers in the same element in XFEM.

Modeling the presence of inclusions and discontinuities in an otherwise uniform domain is utilized by enrichment functions in XFEM. The partition of unity method (PUM), formalized by Babuska and Melenk [23], is the foundation of how to incorporate the enrichment function into the finite element framework. In PUM, the enrichment is computed as the product of the enrichment function and the standard shape functions that satisfy the partition of unity property for the enrichment. The enrichment functions are known a-priori and represent the response well around inclusions or a crack.

We consider the following discretization of the displacement field for a domain reinforced by one or multiple short fiber inclusions:

$$
\mathbf{u}(\mathbf{x}, t)=\sum_{a=1}^{n_{n}} N_{a}(\mathbf{x}) \hat{\mathbf{u}}_{a}+\sum_{\alpha=1}^{n}\left[\sum_{b=1}^{n_{\mathrm{en}}^{\alpha}} N_{\mathcal{I}_{b}^{\alpha}}(\mathbf{x}) \psi_{\alpha}(\mathbf{x}) \hat{\mathbf{c}}_{b \alpha}\right]+\sum_{\alpha=1}^{n}\left[\sum_{c=1}^{n_{\mathrm{en}}^{\alpha}} N_{\mathcal{I}_{c}^{\alpha}}(\mathbf{x}) \Upsilon_{\alpha}(\mathbf{x}) \hat{\mathbf{d}}_{c \alpha}\right]
$$

where, $\mathbf{u}$ denotes the displacement field; $\mathbf{x}$ and $t$ are the space and time coordinates, respec- 
tively; $n_{n}$ the total number of mesh nodes in the finite element discretization; $n$ is the number of fibers, $n_{\mathrm{en}}^{\alpha}$ is the number of enriched nodes for fiber $\alpha ; N_{a}$, the standard finite element shape function associated with node $a ; \hat{\mathbf{u}}_{a}, \hat{\mathbf{c}}_{b \alpha}$ and $\hat{\mathbf{d}}_{c \alpha}$ the nodal coefficients of the standard, fiber enrichment and debonding enrichments for each fiber $\alpha$, respectively; $\mathcal{I}^{\alpha}$ is the index set of enriched nodes for fiber $\alpha ; \mathcal{I}_{a}^{\alpha} \in \mathcal{I}^{\alpha}$ the index of an enriched node, $a$; the fiber enrichment function and the debonding enrichment function are different for each fiber, $\alpha$, denoted as $\psi_{\alpha}$ and $\Upsilon_{\alpha}$, respectively.

The standard finite element approximation of the response field corresponds to the first right hand side term in Eq. 1. The second term represents the presence of the fiber within the domain, accounting for the strain discontinuity in the approximation space and is a function of the fiber enrichment, $\psi_{\alpha}$. The displacement jump due to the progressive loss of the cohesive bond between the fiber and the matrix is represented in the third term and is a function of the debonding enrichment function, $\Upsilon_{\alpha}$. In elements with multiple fibers, approximation of both the fiber strain and debonding discontinuities are captured for each fiber individually. Therefore, there is separate set of nodal coefficients for each fiber enrichment.

\subsection{Fiber enrichment function}

The enrichment functions for the fiber enrichment and debonding enrichment for the high aspect ratio short fiber inclusions have been previously proposed by the authors [53, 54] and are briefly discussed here.

The reinforcing fiber is taken to be straight with a high aspect ratio and is entirely embedded in the open bounded domain of the composite body, $\Omega$, where $\Omega \subset \mathbb{R}^{2}$. The domain of the fiber is therefore approximated as a line segment.

The level set associated with the domain of the fiber, $\phi_{c}(\mathbf{x})$, is expressed as:

$$
\phi_{c}(\mathbf{x})=\|\mathbf{x}-\mathcal{P}(\mathbf{x})\|
$$

$\phi_{c}$ divides the domain of the body along the plane of the fiber with positive values on each side and has zero value along the fiber. The tips of a fiber are identified using:

$$
\phi_{\lambda}(\mathbf{x})=\left(\mathbf{x}-\mathbf{x}_{\lambda}\right) \cdot \mathbf{t}_{\lambda} ; \quad \lambda=1,2
$$

$\phi_{\lambda}$ provides the zero level set along the plane normal to the fiber passing through the fiber tip. $\phi_{\lambda}$ is positive on one side of the domain cut by the zero level set, and negative elsewhere within the composite body. $\mathcal{P}(\mathbf{x})$ is the projection of $\mathbf{x}$ onto the fiber, $\mathbf{x}_{\lambda}$ is the position of the fiber tip, and $\mathbf{t}_{\lambda}$ denotes the tangent at the fiber tip, $\lambda$ (i.e., $\mathbf{t}_{1}=\left(\mathbf{x}_{1}-\mathbf{x}_{2}\right) / l$ and $\left.\mathbf{t}_{2}=\left(\mathbf{x}_{2}-\mathbf{x}_{1}\right) / l=-\mathbf{t}_{1}\right)$; and $l=\left\|\mathbf{x}_{2}-\mathbf{x}_{1}\right\|$ is the length of the fiber. 


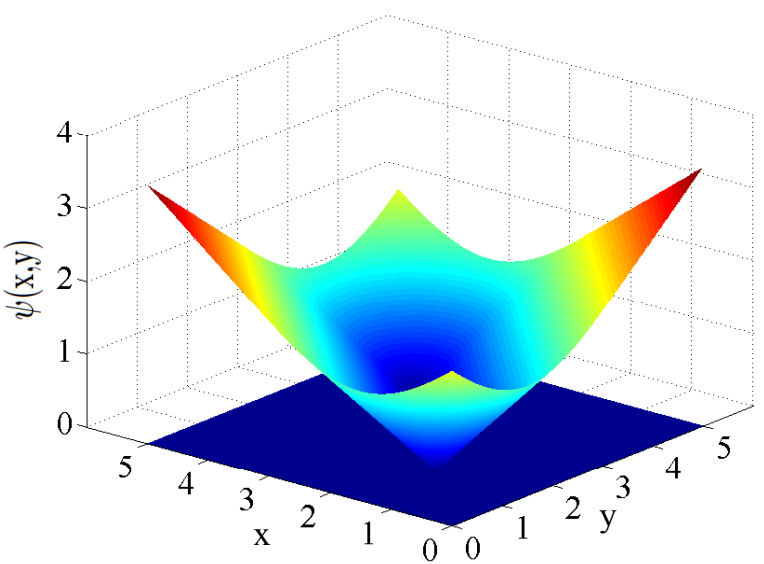

(a)

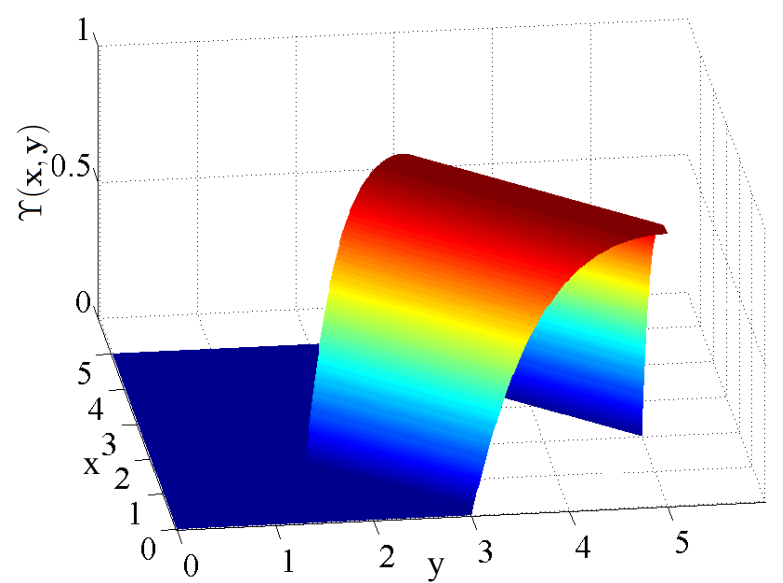

(b)

Figure 2: Three dimensional view of enrichment functions: (a) short fiber enrichment function; and (b) debonding enrichment function. [54]

Using the level set functions from Eqs. 2 and 3, the enrichment function for the fiber is expressed as:

$$
\psi_{\alpha}(\mathbf{x})=\left[\prod_{\lambda=1}^{2} H\left(-\phi_{\lambda}\right)\right] \phi_{c}(\mathbf{x})+\sum_{\lambda=1}^{2} H\left(\phi_{\lambda}\right) d_{\lambda}(\mathbf{x})
$$

where, $H$ denotes the Heaviside function; and $d_{\lambda}(\mathbf{x})=\left\|\mathbf{x}-\mathbf{x}_{\lambda}\right\|$ denotes the distance to the fiber tip.

The enrichment function is illustrated in Fig. 2a. Enrichment functions with similar Vshaped enrichments have been employed in inclusion problems (e.g., [56]), with the exception of the treatment of the tip conditions. The inclusion of $\psi_{\alpha}(\mathbf{x})$ in the discretization of the displacement field incorporates a strain discontinuity mode along the fiber position and the displacements around the fiber can therefore be accurately captured without explicitly discretizing the fiber domain. The form of Eq. 4 for the enrichment function ensures that the approximation basis captures the strain discontinuity but stays smooth otherwise around the sides and tips of the fiber. Except for the domain of the fiber, the enrichment function is nonzero everywhere in the composite domain. We consider the enrichment around a small domain around the fiber and employ standard finite element shape functions in the remainder of the problem domain.

\subsection{Debonding enrichment function}

The debonding enrichment function $\Upsilon_{\alpha}$, is defined using the fiber domain and tip level set functions, similar to the fiber enrichment function. In contrast to the fiber enrichment, the debonding enrichment function introduces a discontinuity in the displacement field.

To mimic the shape of the fiber-matrix debonding, the shape of the debonding enrichment 
function is governed by the discontinuity function, $\phi_{p}$ :

$$
\phi_{p}(\mathbf{x})=1+\frac{\tan \theta}{2} s(\mathbf{x})^{2}\left(1-s(\mathbf{x})^{2}\right)-s(\mathbf{x})^{2}\left(2-s(\mathbf{x})^{2}\right)
$$

in which, $s(\mathbf{x})$ is the position along the length of the fiber, where $-1 \leq s \leq 1$ and $\theta$ is the slope of the discontinuity at the tips of the fiber. $\phi_{p}$ is taken to be a fourth order polynomial, where the polynomial is constrained with the following assumptions: (1) The ends of the fiber are taken to remain fully attached to the matrix; (2) Maximum debonding occurs at the center of the fiber; and (3) The function is normalized such that the maximum value is unity at the center of the fiber.

The slope of the discontinuity at the tips of the fiber, $\theta$, controls the shape of the discontinuity function:

$$
\theta=\tan ^{-1}\left(\left.\frac{d \phi_{p}}{d s}\right|_{s=-1}\right)
$$

The discontinuity curve displays inflection points, when $\theta$ is less than a threshold value (i.e., $\theta_{\mathrm{th}}$ ), occurring along the length of the fiber at positions that depend on the value of $\theta$. The discontinuity curve is convex above the threshold value, in which the threshold value for the chosen function form (Eq. 5) is $\theta_{\mathrm{th}}=58^{\circ}$. In the numerical verification studies provided in this manuscript, the shape parameter is set to $\theta=81^{\circ}$.

The debonding enrichment function for the fiber is then expressed in terms of the discontinuity functions, $\phi_{\lambda}(\mathbf{x})$ and $\phi_{c}(\mathbf{x})$ as:

$$
\Upsilon_{\alpha}(\mathbf{x})=\phi_{p} H\left(r\left(\phi_{c}\right)\right)\left(\prod_{\lambda=1}^{2} H\left(-\phi_{\lambda}\right)\right)
$$

where $r= \pm \phi_{c}$ is the signed distance function. Fig. 2b illustrates a three dimensional visualization of the debonding enrichment function.

The choice for the shape of the fiber-matrix debonding (i.e., the enrichment function) is based on observations from numerous evaluations of direct finite element simulations of a short fiber inclusion subjected to remote tensile stress. This enrichment is employed to capture debonding along both normal and tangential directions. Interfacial damage in directions normal and tangential to fibers has been observed experimentally in fiber reinforced concrete composites [29]. In this manuscript, we investigate the effect of presence of nearby fibers on fiber-matrix debonding, which leads to complex traction patterns along the fiber-matrix interface. The proposed parabolic and symmetric enrichment function used in the context of XFEM have the ability to capture asymmetric and complex debonding patterns as demonstrated below. 


\section{Governing Equations and Model Formulation}

The mechanical equilibrium within the domain for a composite with randomly oriented short fibers is expressed as:

$$
\nabla \cdot \boldsymbol{\sigma}(\mathbf{x}, t)=0 ; \quad \mathbf{x} \in \Omega
$$

and;

$$
\boldsymbol{\sigma}=\mathbf{L}: \boldsymbol{\epsilon}(\mathbf{x}, t)
$$

where, $\boldsymbol{\sigma}$ is the stress tensor; $\nabla(\cdot)$ the divergence operator; and $\boldsymbol{\epsilon}$ denotes the strain tensor. The strain is taken to be the symmetric gradient of the displacement field $\left(\boldsymbol{\epsilon}=\nabla^{s} \mathbf{u}\right)$. L denotes the tensor of elastic moduli, taken to be symmetric and positive definite. All fibers, as well as the matrix, are assumed to remain elastic under the applied loading and only quasi-static response is considered.

The exterior boundary conditions are expressed as:

$$
\begin{array}{cc}
\mathbf{u}(\mathbf{x}, t) & =\tilde{\mathbf{u}}(\mathbf{x}, t) ; \quad \mathbf{x} \in \Gamma_{u} \\
\boldsymbol{\sigma} \cdot \boldsymbol{n} & =\tilde{\mathbf{t}}(\mathbf{x}, t) ; \quad \mathbf{x} \in \Gamma_{t}
\end{array}
$$

in which, $\tilde{\mathbf{u}}$ and $\tilde{\mathbf{t}}$ are the prescribed boundary displacements and tractions defined on boundaries $\Gamma_{u}$ and $\Gamma_{t}$, respectively, such that $\Gamma_{u} \cap \Gamma_{t}=\emptyset$ and $\partial \Omega=\Gamma_{u} \cup \Gamma_{t}$. The domains of fiber $\alpha$, and the matrix are denoted as $\Omega_{\alpha}$ and $\Omega_{m}$, respectively. Fibers are taken to be fully embedded in the matrix and do not intersect with exterior boundaries or with each other (i.e., $\Omega=\Omega_{m} \cup \bigcup_{\alpha=1}^{n} \Omega_{\alpha}$ ), but are able to neighbor closely to each other.

The traction continuity across the fiber-matrix interface is given as:

$$
\llbracket \boldsymbol{T} \rrbracket=\llbracket \boldsymbol{\sigma} \cdot \boldsymbol{n} \rrbracket=0 \quad \mathbf{x} \in \Gamma_{\alpha} \equiv \partial \Omega_{m} \cap \partial \Omega_{\alpha} \quad \forall \alpha
$$

in which the traction $\boldsymbol{T}$, is a function of the normal and tangential tractions $\left(\boldsymbol{T}=\boldsymbol{T}\left(T_{n}, T_{t}\right)\right.$; $\boldsymbol{n}$ is the outward unit vector to a boundary; and $\llbracket \cdot \rrbracket$ is the jump operator. $\Gamma_{\alpha}$ denotes the interface between the fiber, $\alpha$ and the matrix.

The physical deterioration occurring at the interface is represented by the cohesive zone law describing the relationship between surface traction and separation. A bilinear cohesive law is considered in this study. The uncoupled normal and tangential tractions are expressed as:

$$
T_{n}\left(\llbracket \mathrm{u}_{n} \rrbracket\right)= \begin{cases}\frac{\llbracket \mathrm{u}_{n} \rrbracket}{d_{n}} \sigma_{\max } & d_{n} \geq \llbracket \mathrm{u}_{n} \rrbracket \geq 0 \\ \frac{\sigma_{\max }}{d_{n}^{\text {crit }}-d_{n}}\left(d_{n}^{\text {crit }}-\llbracket \mathrm{u}_{n} \rrbracket\right) & d_{n}^{\text {crit }} \geq \llbracket \mathrm{u}_{n} \rrbracket \geq d_{n} \\ 0 & \llbracket \mathrm{u}_{n} \rrbracket \geq d_{n}^{\text {crit }}\end{cases}
$$




$$
T_{t}\left(\llbracket \mathrm{u}_{t} \rrbracket\right)= \begin{cases}\frac{\llbracket \mathrm{u}_{t} \rrbracket}{d_{t}} \tau_{\max } & d_{t} \geq \llbracket \mathrm{u}_{t} \rrbracket \geq 0 \\ \frac{\tau_{\max }}{d_{t}^{\text {crit }}-d_{t}}\left(d_{t}^{\text {crit }}-\llbracket \mathrm{u}_{t} \rrbracket\right) & d_{t}^{\text {crit }} \geq \llbracket \mathrm{u}_{t} \rrbracket \geq d_{t} \\ 0 & \llbracket \mathrm{u}_{t} \rrbracket \geq d_{t}^{\text {crit }}\end{cases}
$$

in which, $\llbracket \mathrm{u}_{n} \rrbracket$ and $\llbracket \mathrm{u}_{t} \rrbracket$ are the components of the displacement jump vector (i.e., separation) along the normal and tangential directions, respectively; $d_{n}$ and $d_{t}$ the normal and tangential cohesive characteristic separation lengths, respectively; $\sigma_{\max }$ and $\tau_{\max }$ denote the ultimate normal and tangential tractions, respectively; $d_{n}^{\text {crit }}$ and $d_{t}^{\text {crit }}$ are the maximum normal and tangential displacement jumps, respectively. This manuscript focuses on cases, where the composite is subjected to tensile loading. In cases of compression or shear loading, it is necessary to explicitly impose the impenetrability condition (i.e., $\llbracket u_{n} \rrbracket \geq 0$ ) in the cohesive law as well.

In a two dimensional domain, consider a matrix reinforced by $n$ straight fibers, with length and the thickness of a fiber, $\alpha$, denoted as $l_{\alpha}$ and $t_{\alpha}$, respectively, at an angle, $\theta_{\alpha}$ from the horizontal $(\alpha=1,2, \ldots, n)$, where the fibers are randomly distributed within the domain. In this manuscript, the fiber aspect ratios are taken to be small (i.e., $t_{\alpha} / l_{\alpha} \ll 1$ ). Using the standard procedure, the weak form of Eqs. 8-12 is expressed as follows:

$$
\int_{\Omega_{m}} \boldsymbol{\sigma}: \delta \boldsymbol{\epsilon} d \Omega+\sum_{\alpha=1}^{n} \int_{\Omega_{\alpha}} \boldsymbol{\sigma}: \delta \boldsymbol{\epsilon} d \Omega+\sum_{\alpha=1}^{n} \int_{\Gamma_{\alpha}} \boldsymbol{T} \cdot \delta \llbracket \mathbf{u} \rrbracket d \Gamma-\int_{\Gamma_{t}} \tilde{\mathbf{t}} \cdot \delta \mathbf{u} d \Gamma=0
$$

where, $\delta \mathbf{u}$ denotes the test function; and $\delta \boldsymbol{\epsilon}$ the gradient of the test function.

The stress that develops in the fiber is axial, due to the assumption that high aspect ratio fibers that are embedded in the domain are assumed to have uniform tractions along the fiber. This assumption is verified using direct finite element simulations where the fiber is resolved with highly resolved meshes. No significant shear stress or bending moment develops within the domain of the fiber. The axial stress is expressed as:

$$
\boldsymbol{\sigma}=\sigma_{f}^{\alpha}(s) \boldsymbol{t}_{\alpha} \otimes \boldsymbol{t}_{\alpha}
$$

where the second term in Eq. 15 becomes:

$$
\int_{\Omega_{\alpha}} \boldsymbol{\sigma}: \delta \boldsymbol{\epsilon} d \Omega \simeq t_{\alpha} \int_{\Omega_{\alpha}} \sigma_{f}^{\alpha} \delta \epsilon_{f}^{\alpha} d \Omega
$$

where, $\delta \epsilon_{f}^{\alpha}=\delta \boldsymbol{\epsilon}: \boldsymbol{t}_{\alpha} \otimes \boldsymbol{t}_{\alpha}$. The axial stress in fiber $\alpha$, is taken to be proportional to the axial strain (i.e., $\sigma_{f}^{\alpha}=E_{f} \epsilon_{f}^{\alpha}$ ), where $E_{f}$ is the elastic modulus of the fiber. Upon complete debonding between the fiber and the matrix, bending of the fiber may also develop. This 
deformation mode is not accounted for in the current manuscript.

Under the condition that aspect ratios of the fibers are very high, we assume that tractions along the two opposing faces of a fiber in the thickness direction are uniform:

$$
\left.\boldsymbol{T}^{+}(s) \cdot \boldsymbol{n}^{+}\right|_{\Gamma_{\alpha}^{+}}-\left.\boldsymbol{T}^{-}(s) \cdot \boldsymbol{n}^{-}\right|_{\Gamma_{\alpha}^{-}}=0 ;\left.\quad \boldsymbol{T}^{+}(s) \cdot \boldsymbol{t}^{+}\right|_{\Gamma_{\alpha}^{+}}-\left.\boldsymbol{T}^{-}(s) \cdot \boldsymbol{t}^{-}\right|_{\Gamma_{\alpha}^{-}}=0
$$

where the fiber domain is $\Omega_{\alpha}$, and the fiber normal and tangent vectors are, $\boldsymbol{n}_{\alpha}$ and $\boldsymbol{t}_{\alpha}$, respectively. Debonding along the two faces of a fiber would typically occur concurrently for a short fiber embedded in a matrix under the traction conditions. However, the fiber-matrix debonding is likely to initiate at a weak spot at one side of the fiber. Upon complete debonding at the weak side, the tractions along the opposing (unbonded) side relax. In this manuscript, we assume the tips of the fiber remain attached to the matrix. The third term in Eq. 15 models the progressive debonding process between the fiber and the matrix. The internal boundary term then reduces to:

$$
\int_{\Gamma_{\alpha}} \boldsymbol{T} \cdot \delta \llbracket \mathbf{u} \rrbracket d \Gamma=\int_{\Gamma_{\alpha}^{+}} \boldsymbol{T} \cdot \delta \llbracket \mathbf{u} \rrbracket d \Gamma
$$

In the limit where fiber aspect ratios tend to infinity, the weak form of the governing equations is expressed as:

$$
\int_{\Omega} \boldsymbol{\sigma}: \delta \boldsymbol{\epsilon} d \Omega+\sum_{\alpha=1}^{n} t_{\alpha} E_{f} \int_{\Omega_{\alpha}} \epsilon_{f}^{\alpha} \delta \epsilon_{f}^{\alpha} d \Omega+\sum_{\alpha=1}^{n} \int_{\Gamma_{\alpha}} \boldsymbol{T} \cdot \delta \llbracket \mathbf{u} \rrbracket d \Gamma-\int_{\Gamma_{t}} \tilde{\mathbf{t}} \cdot \delta \mathbf{u} d \Gamma=0
$$

The domain of the matrix is taken to occupy the entire domain, since the domains of the fibers are computed are vanishingly small. Therefore, the limits of the integral of the first term in Eq. 15 is set to $\Omega$. The proposed formulation and implementation is limited to 2-D. While the general ideas remain relevant for the $3-\mathrm{D}$ case, the $3-\mathrm{D}$ implementation poses non-trivial challenges, and beyond the scope of this manuscript.

\section{Computational Formulation and Implementation}

We employ the extended finite element method to discretize and evaluate the governing equations in Eqs. 8-12, and Eqs. 15-20. Matrix notation is employed in the formulations for convenience. The weak form of the governing equation (Eq. 20) is written in the matrix form as:

$$
\int_{\Omega} \delta \boldsymbol{\epsilon}^{T} \boldsymbol{\sigma} d \Omega-\int_{\Gamma_{t}} \delta \mathbf{u}^{T} \tilde{\mathbf{t}} d \Gamma+\sum_{\alpha=1}^{n} t_{\alpha} E_{f} \int_{\Omega_{\alpha}} \epsilon_{f}^{\alpha} \delta \epsilon_{f}^{\alpha} d \Omega+\sum_{\alpha=1}^{n} \int_{\Gamma_{\alpha}} \delta \llbracket \mathbf{u} \rrbracket^{T} \boldsymbol{T} d \Gamma=0
$$

in which, the superscript $T$ indicates transpose. 
Using the Bubnov-Galerkin approach, the discretization of the displacement field follows Eq. 1 and the discretization of the test function uses the same shape functions as the trial function. The first term in Eq. 21 at the element level integral becomes:

$$
\int_{\Omega} \delta \boldsymbol{\epsilon}^{T} \boldsymbol{\sigma} d \Omega=\sum_{e=1}^{n_{e}}\left(\mathbf{V}^{e}\right)^{T} \int_{\Omega_{e}}\left(\mathbf{B}^{e}\right)^{T} \boldsymbol{\sigma}\left(\mathbf{U}^{e}\right) d \Omega
$$

where, $n_{e}$ is the total number of elements discretizing the domain; and $\Omega_{e}$ is the domain of the element, $e$. The nodal coefficient vectors of the trial and test functions in element $e$, are $\mathbf{U}^{e}$ and $\mathbf{V}^{e}$, respectively, are expressed as:

$$
\mathbf{U}^{e}=\left\{\hat{\mathbf{u}}^{e} ; \hat{\mathbf{c}}^{e} ; \hat{\mathbf{d}}^{e}\right\} ; \quad \mathbf{V}^{e}=\left\{\delta \hat{\mathbf{u}}^{e} ; \delta \hat{\mathbf{c}}^{e} ; \delta \hat{\mathbf{d}}^{e}\right\}
$$

in which, a semicolon implies that the construction forms a column vector.

The standard fiber enrichment and the jump enrichment degrees of freedom correspond to the three components of the nodal coefficient vectors, respectively, and are expressed as:

$$
\hat{\mathbf{u}}^{e}=\left\{\hat{\mathbf{u}}_{1}^{e} ; \hat{\mathbf{u}}_{2}^{e} ; \ldots ; \hat{\mathbf{u}}_{n_{n}^{e}}^{e}\right\} \quad \hat{\mathbf{c}}^{e}=\left\{\hat{\mathbf{c}}_{1}^{e} ; \hat{\mathbf{c}}_{2}^{e} ; \ldots ; \hat{\mathbf{c}}_{n_{\mathrm{en}}^{e}}^{e}\right\} \quad \hat{\mathbf{d}}^{e}=\left\{\hat{\mathbf{d}}_{1}^{e} ; \hat{\mathbf{d}}_{2}^{e} ; \ldots ; \hat{\mathbf{d}}_{n_{\mathrm{en}}^{e}}^{e}\right\}
$$

where, $\hat{\mathbf{u}}_{a}^{e}, \hat{\mathbf{c}}_{a}^{e}$ and $\hat{\mathbf{d}}_{a}^{e}$ are the vectors of unknown coefficients for standard and enriched degrees of freedom at element, $e$ and node $a$; and $n_{n}^{e}$ and $n_{\mathrm{en}}^{e}$ are the number of standard and enriched nodes within element, $e$, respectively. The components of $\mathbf{V}^{e}$ are similarly defined.

The size of $\hat{\mathbf{c}}^{e}$ and $\hat{\mathbf{d}}^{e}$ vectors may differ for each element, and depends on the number of fiber enrichments included in the element as well as whether the enrichment for each fiber is full or partial as described below.

The gradient vector $\mathbf{B}^{e}$, in Eq. 22 is expressed as:

$$
\mathbf{B}^{e}=\left\{\hat{\mathbf{B}}_{1}^{e}, \hat{\mathbf{B}}_{2}^{e}, \ldots, \hat{\mathbf{B}}_{n_{n}^{e}}^{e}, \overline{\mathbf{B}}_{1}^{e}, \overline{\mathbf{B}}_{2}^{e}, \ldots, \overline{\mathbf{B}}_{n_{\mathrm{en}}^{e}}^{e}, \tilde{\mathbf{B}}_{1}^{e}, \tilde{\mathbf{B}}_{2}^{e}, \ldots, \tilde{\mathbf{B}}_{n_{\mathrm{en}}^{e}}^{e}\right\}
$$

in which, the gradient terms are:

$$
\hat{\mathbf{B}}_{a}^{e}=\left[\begin{array}{cc}
N_{a, x}^{e} & 0 \\
0 & N_{a, y}^{e} \\
N_{a, y}^{e} & N_{a, x}^{e}
\end{array}\right] ; \overline{\mathbf{B}}_{a}^{e}=\left[\begin{array}{cc}
\left(N_{a}^{e} \psi_{\alpha}\right)_{, x} & 0 \\
0 & \left(N_{a}^{e} \psi_{\alpha}\right)_{, y} \\
\left(N_{a}^{e} \psi_{\alpha}\right)_{, y} & \left(N_{a}^{e} \psi_{\alpha}\right)_{, x}
\end{array}\right] ; \tilde{\mathbf{B}}_{a}^{e}=\left[\begin{array}{cc}
\left(N_{a}^{e} \Upsilon_{\alpha}\right)_{, x} & 0 \\
0 & \left(N_{a}^{e} \Upsilon_{\alpha}\right)_{, y} \\
\left(N_{a}^{e} \Upsilon_{\alpha}\right)_{, y} & \left(N_{a}^{e} \Upsilon_{\alpha}\right)_{, x}
\end{array}\right]
$$

where, differentiation is indicated by a subscript followed by a comma. The first term in Eq. 21 is then written in the matrix form as:

$$
\mathbf{V}^{T} \mathbf{f}_{\text {int }, 1}(\mathbf{U})
$$


in which, the internal force component is obtained by assembling the corresponding element matrices:

$$
\mathbf{f}_{\mathrm{int}, 1}(\mathbf{U})=\mathbf{A}_{e=1}^{n_{e}} \int_{\Omega_{e}}\left(\mathbf{B}^{e}\right)^{T} \boldsymbol{\sigma}\left(\mathbf{U}^{e}\right) d \Omega
$$

$\mathbf{U}$ and $\mathbf{V}$ are obtained by assembling the corresponding element vectors.

The external force contribution from Eq. 21 is formulated by decomposing the boundary integral into its elemental components:

$$
\int_{\Gamma_{t}} \delta \mathbf{u}^{T} \tilde{\mathbf{t}} d \Gamma=\mathbf{V}^{T} \mathbf{f}_{\mathrm{ext}}
$$

The external force vector is obtained through the assembly of the elemental contributions:

$$
\mathbf{f}_{\mathrm{ext}}^{e}=\int_{\Gamma_{t}^{e}} \mathbf{f}^{e}(\mathbf{x}) d \Gamma ; \quad e \in \mathcal{I}^{t}
$$

$\mathcal{I}^{t}$ denotes the index set of elements along the traction boundary, $\Gamma_{t} ; \Gamma_{t}^{e}$ denotes the part of the traction boundary approximated by element $e$; and where the force vector is defined as:

$$
\mathbf{f}^{e}=\left\{\hat{\mathbf{f}}_{1}^{e} ; \hat{\mathbf{f}}_{2}^{e} ; \ldots ; \hat{\mathbf{f}}_{n_{n}^{e}}^{e} ; \overline{\mathbf{f}}_{1}^{e} ; \overline{\mathbf{f}}_{2}^{e} ; \ldots ; \overline{\mathbf{f}}_{n_{\mathrm{en}}^{e}}^{e} ; \tilde{\mathbf{f}}_{1}^{e} ; \tilde{\mathbf{f}}_{2}^{e} ; \ldots ; \tilde{\mathbf{f}}_{n_{\mathrm{en}}^{e}}^{e}\right\}
$$

where, the components of the element force vector are expressed as:

$$
\hat{\mathbf{f}}_{a}^{e}(\mathbf{x})=N_{a}^{e}(\mathbf{x}) \tilde{\mathbf{t}}(\mathbf{x}) ; \quad \overline{\mathbf{f}}_{a}^{e}(\mathbf{x})=\hat{\mathbf{f}}_{a}^{e}(\mathbf{x}) \psi_{\alpha}(\mathbf{x}) ; \quad \tilde{\mathbf{f}}_{a}^{e}(\mathbf{x})=\hat{\mathbf{f}}_{a}^{e}(\mathbf{x}) \Upsilon_{\alpha}(\mathbf{x})
$$

The deformation of the fibers are accounted for in the third term in Eq. 21. The fiber components that lie in enriched elements are expressed in the integral term as:

$$
\int_{\Omega_{\alpha}} \epsilon_{f}^{\alpha} \delta \epsilon_{f}^{\alpha} d \Omega=\sum_{e=1}^{n_{e}^{\alpha}} \int_{\Omega_{\alpha}^{e}} \epsilon_{f}^{\alpha} \delta \epsilon_{f}^{\alpha} d \Omega
$$

in which, $n_{e}^{\alpha}$ denotes the number of fully enriched elements that contains a part of the fiber, $\alpha$. The fiber is assumed to deform uniformly within each element, which leads to:

$$
\begin{aligned}
t_{\alpha} E_{f} \int_{\Omega_{\alpha}^{e}} \epsilon_{f}^{\alpha} \delta \epsilon_{f}^{\alpha} d \Omega & =\frac{t_{\alpha} E_{f}}{l_{\alpha e}}\left[\left(\mathbf{u}\left(\mathbf{x}_{2}^{\alpha e}\right)-\mathbf{u}\left(\mathbf{x}_{1}^{\alpha e}\right)\right) \cdot \boldsymbol{t}_{\alpha}\right]\left[\left(\delta \mathbf{u}\left(\mathbf{x}_{2}^{\alpha e}\right)-\delta \mathbf{u}\left(\mathbf{x}_{1}^{\alpha e}\right)\right) \cdot \boldsymbol{t}_{\alpha}\right] \\
& =\left(\delta \hat{\mathbf{u}}^{e}\right)^{T} \breve{\mathbf{K}}_{e}^{\alpha s} \hat{\mathbf{u}}^{e}
\end{aligned}
$$

where, $\mathbf{x}_{1}^{\alpha e}$ and $\mathbf{x}_{2}^{\alpha e}$ are defined as the entry and exit positions of the fiber on the enriched element, respectively. The fiber entry and exit positions are on the element edges, when the fiber crosses the domain of the element. If the domain of the fiber ends within the element, the end position of the fiber segment coincides with the fiber tip. The length of the fiber segment 
that lies within the element is denoted as $l_{\alpha e}=\left\|\mathbf{x}_{2}^{\alpha e}-\mathbf{x}_{1}^{\alpha e}\right\| . \boldsymbol{t}_{\alpha}$ is the tangent vector on the fiber domain.

The enrichment functions vanish on the domain of the fiber and therefore the stiffness matrix is nonzero only for the standard degrees of freedom:

$$
\breve{\mathbf{K}}_{e}^{\alpha}=\left[\begin{array}{cc}
\breve{\mathbf{K}}_{e}^{\alpha s} & \mathbf{0} \\
\mathbf{0} & \mathbf{0}
\end{array}\right]
$$

The contribution can be computed using the standard assembly operation:

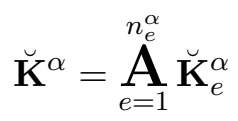

The internal contribution from the second term in Eq. 21 then becomes:

$$
\mathbf{f}_{\text {int }, 2}^{\alpha}(\mathbf{U})=\breve{\mathbf{K}}^{\alpha} \mathbf{U}
$$

The progressive debonding between the fiber and the matrix is accounted for in the fourth component of Eq. 21. The progressive debonding is expressed in terms of the jump enrichment degrees of freedom. For an arbitrary fiber, $\alpha$ :

$$
\int_{\Gamma_{\alpha}}(\delta \llbracket \mathbf{u} \rrbracket)^{T} \boldsymbol{T}(\llbracket \mathbf{u} \rrbracket) d \Gamma=(\delta \hat{\mathbf{d}})^{T} \int_{\Gamma_{\alpha}}\left(\mathbf{P}^{\alpha}\right)^{T} \boldsymbol{T}(\hat{\mathbf{d}}) d \Gamma=(\delta \hat{\mathbf{d}})^{T} \mathbf{f}_{\mathrm{int}, 3}^{d \alpha}(\hat{\mathbf{d}})
$$

in which, shape functions for the jump enrichments are included in $\mathbf{P}^{\alpha}$. A force vector contribution is assembled from the debonding enrichment terms (i.e., $\mathbf{V}^{T} \mathbf{f}_{\text {int }, 3}^{\alpha}(\mathbf{U})$ ):

$$
\mathbf{f}_{\text {int }, 3}^{\alpha}=\left\{\mathbf{0} ; \mathbf{0} ; \mathbf{f}_{\text {int }, 3}^{d \alpha}\right\}
$$

where the internal force contribution is only due to the jump degrees of freedom. A system of nonlinear equations from equilibrium of the three internal force and external force contributions is expressed in the form of:

$$
\phi(\mathbf{U})=\mathbf{f}_{\text {int }}(\mathbf{U})-\mathbf{f}_{\text {ext }}=0
$$

where,

$$
\mathbf{f}_{\mathrm{int}}(\mathbf{U})=\mathbf{f}_{\mathrm{int}, 1}+\sum_{\alpha=1}^{n}\left(\mathbf{f}_{\mathrm{int}, 2}^{\alpha}+\mathbf{f}_{\mathrm{int}, 3}^{\alpha}\right)
$$

The Newton-Raphson method is used to incrementally evaluate the nonlinear system in Eq. 41. 


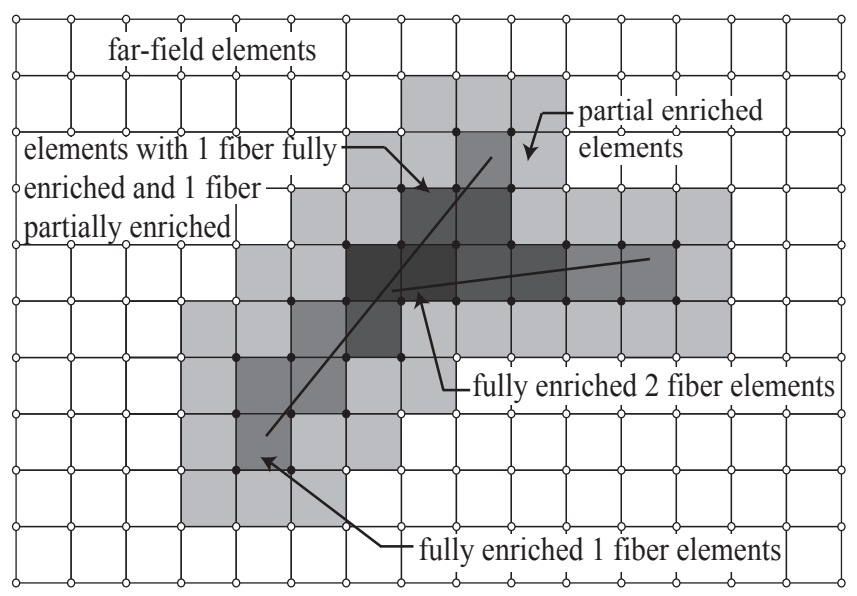

Figure 3: Decomposition of the problem domain into subdomains of far-field elements approximated by standard basis, partially and fully enriched elements for two fibers occupying the same elements in a domain.

\subsection{Numerical integration}

The numerical integration of enriched elements differs for various element types depending on the configuration of the inclusions that lie within them. In XFEM, the extent of the subdomain around an inclusion that is enriched is chosen either based on the geometry or the discretization. For modeling cracks, a geometry-based approach is considered, typically using specified a radius around the crack tip. The stress fields around the crack tip vary as a function of the distance from the tip. In this study, the enrichment domain is chosen based on the discretization since the enrichments functions accurately represent the local behavior around the inclusion.

The domain consists of four different element types as illustrated in Fig. 3: (1) Far field elements with no enrichment; (2) elements with partial enrichment from one or multiple fibers; (3) fully enriched elements crossed by one or multiple fibers; and (4) fully enriched elements crossed by fibers and partially enriched by additional fibers.

The integration rules for the various element types are as follows:

1. Far field elements: Elements have no enrichment. Since no additional functions are employed, the element integration is performed using the standard quadrature rules.

2. Partially enriched elements: Some nodes include enrichment from one or multiple fiber inclusions but no intra-element strain or displacement discontinuity exists. Standard integration is employed. Higher order integration rules could increase the accuracy, but are not employed for efficiency.

3. Fully enriched elements that contain one or multiple fiber inclusions: The elements take into consideration the amount and location of the fiber inclusions rather than integrating 




Figure 4: Integration of elements with multiple fibers.

the elements for each inclusion separately. The elements are either split by the fibers or contain fiber tips (see Fig. 4). For the split fiber, Delaunay triangulation is used to decompose each split part into triangular sub-elements. For fiber tip elements, the elements are split along the normal direction at the fiber tip and along the fiber direction and Delaunay triangulation is used to decompose each split part into triangular subelements. The element splitting at the fiber normal ensures that the components of the enrichment function that pertain to the fiber tip and fiber level sets are integrated separately. For both cases, high order integration rules are used in each sub-element.

4. Fully enriched elements that contain a fiber inclusion and are partially enriched by additional fibers: The elements use the same element splitting rules as in the full enriched element type, and use higher order integration rules for each sub element. The element is treated for the full enrichment of the inclusions in the element as well as for the partial enrichments of the element.

In full enrichment cases, triangular sub-elements aligned with the fiber faces are used in the integration of a 2-D quadrilateral. The triangular sub-elements contain three integration points and use the standard Gauss quadrature rules. The partially enriched elements and the far-field elements use Gauss quadrature rules with four integration points [57]. The partially enriched elements do not have sub elements since the fiber does not cross through the element.

\subsection{Effect of mesh refinement on numerical integration}

When modeling multiple fibers in close proximity, mesh refinement has a significant effect on numerical integration. The change in numerical integration in turn effects the mesh convergence studies as described below and lead to non-monotone convergence.

Figure 5 illustrates two neighboring fibers in a domain discretized using three meshes with 


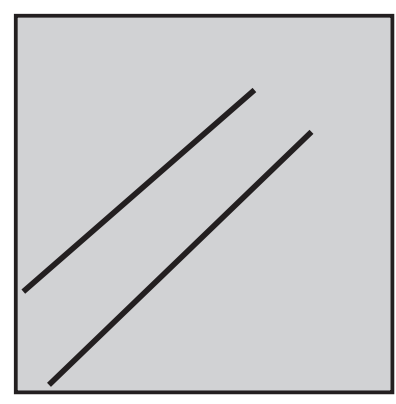

(a)

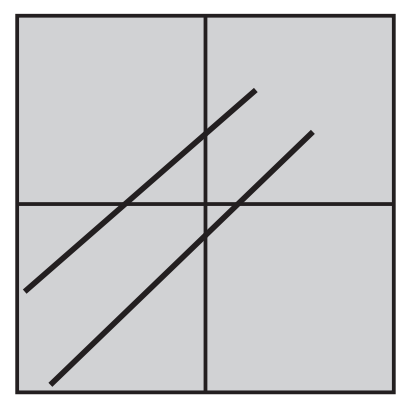

(b)

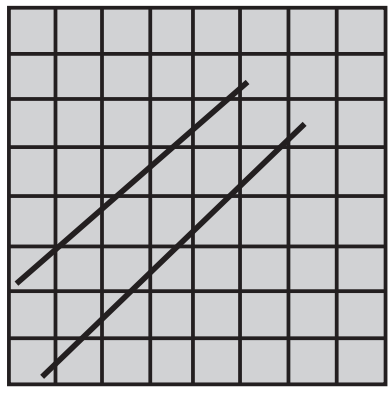

(c)

Figure 5: Change in the integration rules as a function of mesh density: (a) coarse discretization; (b) medium discretization; and (c) fine discretization.

increasing mesh density. In Fig. 5a, a single element encompasses both fibers. When the element size is reduced as in Fig. 5b, two elements are intersected by both fibers, whereas the two other elements are intersected by a single fiber. Figure 5c displays the same fibers when the element size is further decreased. In this case, there are no elements intersected by more than a single fiber. The integration rules for the three cases are quite different from each other and potentially leads to non-monotone convergence.

\subsection{Treatment of partially enriched elements}

In elements that are partially enriched (i.e. elements that have some nodes that are enriched but not all of the nodes), partition of unity no longer holds and the affine transformations (e.g. constant strain modes) cannot be represented exactly. The treatment of these partially enriched elements affect the accuracy and convergence of XFEM models and modified enrichment functions using ramp functions that have local support within the partially enriched element were introduced by Fries [58]. In this manuscript, we apply modified enrichment functions to all nodes of the partially enriched element.

Partially enriched elements could have contributions from a single fiber, or from multiple fibers. When partial enrichment from multiple fibers is present, elements from each fiber are separately considered, similar to the treatment of fully enriched elements when multiple fibers are present in an element. For each fiber, $\alpha$, the modified enrichment functions, $\hat{\psi}_{\alpha}(\mathbf{x})$ and $\hat{\Upsilon}_{\alpha}(\mathbf{x})$, in the partially enriched finite element are denoted as:

$$
\begin{aligned}
& \hat{\psi}_{\alpha}(\mathbf{x})=\sum_{b \in \mathcal{I}_{e}^{\alpha}} N_{b}(\mathbf{x}) \psi_{\alpha}(\mathbf{x}) ; \quad \mathbf{x} \in \Omega_{e} \\
& \hat{\Upsilon}_{\alpha}(\mathbf{x})=\sum_{c \in \mathcal{I}_{e}^{\alpha}} N_{c}(\mathbf{x}) \Upsilon_{\alpha}(\mathbf{x}) ; \quad \mathbf{x} \in \Omega_{e}
\end{aligned}
$$


where, $\mathcal{I}_{e}$ are the nodes in the partially enriched element, $\Omega_{e}$, that are connected to fully enriched elements. The modified enrichment function is active at all nodes of the partially enriched element:

$$
\mathbf{u}^{e}(\mathbf{x})=\sum_{a=1}^{n_{n}^{e}} N_{a}^{e}(\mathbf{x}) \hat{\mathbf{u}}_{a}^{e}+\sum_{\alpha=1}^{n}\left[\sum_{b=1}^{n_{n \alpha}^{e}} N_{b}^{e}(\mathbf{x}) \hat{\psi}_{\alpha}(\mathbf{x}) \hat{\mathbf{c}}_{b \alpha}^{e}\right]+\sum_{\alpha=1}^{n}\left[\sum_{c=1}^{n_{n \alpha}^{e}} N_{c}^{e}(\mathbf{x}) \hat{\Upsilon}_{\alpha}(\mathbf{x}) \hat{\mathbf{d}}_{c \alpha}^{e}\right]
$$

in which, all pertinent variables are defined in the partially enriched element are indicated by the superscript, $e$.

\section{$5 \quad$ Numerical Examples}

In this section, we present numerical examples to demonstrate the performance of the proposed XFEM model in evaluating the response of short fiber reinforced composites with emphasis on the interactions between fibers that lie in the same element. The first example assesses the accuracy characteristics of two fiber inclusion domains embedded in an elastic matrix with perfect interfacial cohesion. The second example illustrates the accuracy characteristics when progressive fiber-matrix debonding is incorporated, including capturing the debonding interaction behavior. The third example investigates the debonding interaction behavior when four fibers are in close proximity of each other and have fibers that lie in the same element. The fourth example displays the case of a domain with a dense population of fibers. Numerical examples demonstrating the performance of a domain reinforced with a single fiber as well as its convergence characteristics are described in Ref. [54].

\subsection{Elastic response of multiple fiber inclusions}

The proposed formulation is verified against the finite element method for an elastic response using a series of simulations of a matrix enriched with two fibers. Four fiber configuration cases were investigated. Each case consists of a domain with two fiber inclusions that have portions of each fiber in close proximity to each other, but they do not touch or overlap. In all cases, the fibers were placed such that there were elements that contained both fibers, for all mesh sizes studied.

The schematic representation of the model problem is shown in Fig. 6a. The size of the domain is $5 \mathrm{~mm}$ by $5 \mathrm{~mm}$ and the fiber length varies between $0.83-2.65 \mathrm{~mm}$. The domain is subjected to uniform displacement controlled tensile loading at the right edge and symmetry boundary conditions are imposed on the left and bottom edges. The matrix material is taken to be concrete with the Young's modulus and Poisson's ratio of the matrix being $14 \mathrm{GPa}$ and 0.3 , respectively. The fibers are taken to be high strength carbon fibers with the Young's 


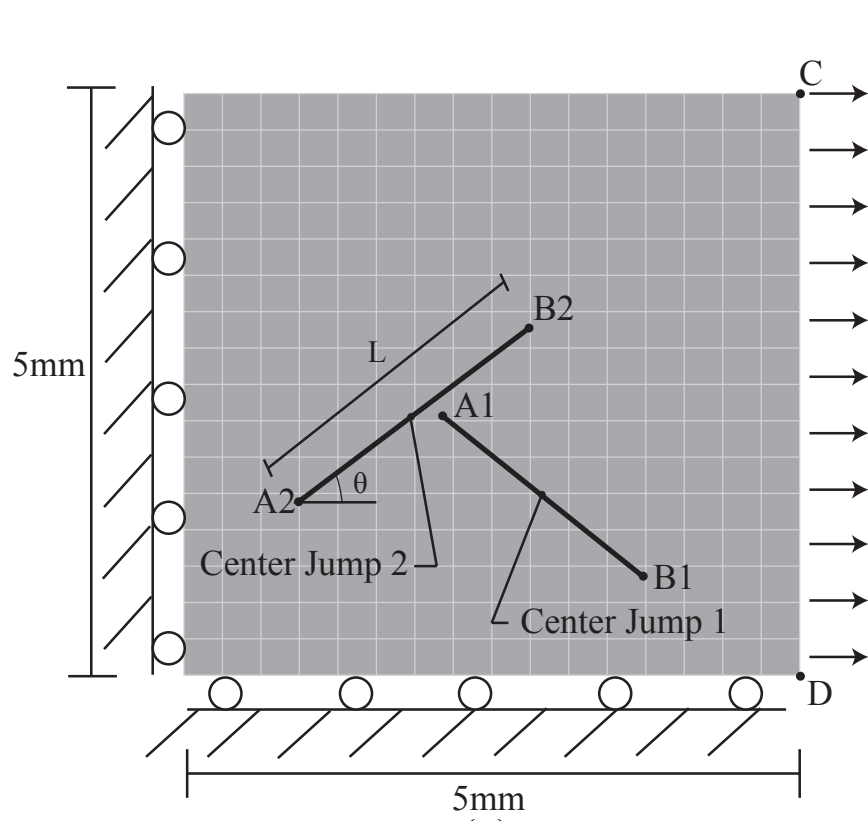

(a)

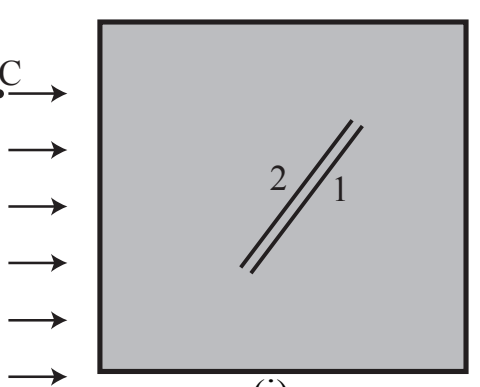

(i)

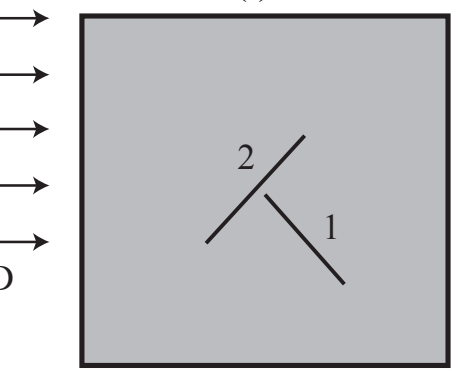

(iii)

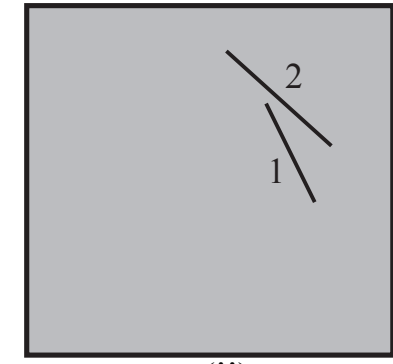

(ii)



(iiii)

(b)

Figure 6: (a) Geometry and boundary conditions of a two fiber inclusion example; and (b) fiber configurations of the four cases studied in the numerical verification: (i) case 1 ; (ii) case 2; (iii) case 3 ; and (iii) case 4 .

modulus and thickness of $207 \mathrm{GPa}$ and $7 \mu \mathrm{m}$, respectively. This example investigates the response of two-dimensional short fiber composites in which the fibers are fully bonded to the matrix. No fiber-matrix debonding occurs and the constituents are taken to deform elastically.

Figure $6 \mathrm{~b}$ illustrates the configurations considered in the verification study. Case 1 consists of two fibers that are parallel to each other with both fibers having a length of $2.65 \mathrm{~mm}$ and angles of 70 degrees measured counterclockwise from the horizontal. They are placed so that fiber 1 is offset slightly to the right of fiber 2 , and the majority of the enriched elements contain enrichments from both fibers. Case 2 contains fibers with lengths of $1.65 \mathrm{~mm}$ and $1.4 \mathrm{~mm}$, with position angles of -20 degrees and -60 degrees, for fiber 1 and fiber 2, respectively. Fiber 2 is placed such that its center is approximately at the left tip of fiber 1 . For case 3 , fiber 1 has a length of $1.37 \mathrm{~mm}$ and a position angle of -45 degrees. Fiber 2 is positioned directly above fiber 1 with a length of $1 \mathrm{~mm}$ and a position angle of 15 degrees. Cases 2 and 3 fiber orientations are placed accordingly to investigate the response of fibers that are randomly oriented but contain elements that have enrichments from multiple fibers. In case 4 , the right tip of fiber 1 and left tip of fiber 2 are placed close to each other. The fibers lengths are $1 \mathrm{~mm}$ and $0.83 \mathrm{~mm}$ and position angles are -23 degrees and 79 degrees for fiber 1 and fiber 2, respectively. The fibers in case 4 are placed so that the fiber tips from each fiber lie in the same element. All fibers are placed such that there are always elements that contain multiple fiber enrichments at each mesh discretization. 
The fibers are idealized as line segments with a thickness and length in the proposed approach. Uniform grids of 1,600 elements up to approximately 62,500 elements with corresponding element sizes of $h=0.125 \mathrm{~mm}$ and $0.02 \mathrm{~mm}$, respectively, were used in the discretization of the domain. The reference model consists of a very fine and non-uniform (to conform to the fiber domains) discretization, in which the fibers are explicitly modeled as two-dimensional solids. The fiber domains are modeled using a very fine grid with an element size of approximately $1 \mu \mathrm{m}$. The reference model discretizations result in approximately 150,000 - 700,000 elements.

Figure 7 shows the absolute point-wise displacement errors of the proposed model with respect to the reference simulation. Point-wise error was computed using the L2-norm for each case and plotted with respect to element size in the domain. Points A1 and A2 correspond to the left tips of fiber 1 and fiber 2, respectively. Points B1 and B2 are the right fiber tips of fiber 1 and fiber 2. Point $\mathrm{C}$ is the upper right corner of the matrix, and point $\mathrm{D}$ is the bottom right corner of the matrix. The 6 different point locations of interest are illustrated in Fig. 6a.

Figure 7 a corresponds to case 1, when two fibers are parallel to each other. The errors showed monotonic convergence from the largest mesh size to the smallest mesh size. The largest error occurred in the left tip of fiber 2, while the lowest errors were in points $\mathrm{C}$ and $\mathrm{D}$ on the domain edge. All errors converged to less than $1 \%$ at the smallest mesh size of $0.02 \mathrm{~mm}$. Case 2 errors are shown in Fig. 7b. Similar to case 1, there is monotonic convergence, with the largest error of the left fiber tip of fiber 2 and the smallest errors at points $\mathrm{C}$ and $\mathrm{D}$. Case 3, shown in Fig. 7c, shows the same trend as in case 1 and case 2 errors. Point A2 however, has a larger error at the largest mesh size than the other cases, but converged to an error of approximately $1.5 \%$ at the smallest mesh size. Fig. 7 d, displays monotonic convergence as well, with a slight variation in point B2 at the smallest mesh size. In all cases, the proposed XFEM model for cases when elements are enriched by multiple fibers is in reasonable agreement with the reference simulation models which used the direct finite element method. As shown in Fig. 7 , in all cases observed, the slope indicates approximately an order of magnitude decrease in error with order of magnitude reduction in element size.

\subsection{Response of multiple fiber inclusions with progressive debond- ing}

In this section, the four cases discussed in Section 5.1 for the elastic response are modeled with progressive debonding to capture the effect of the fiber-matrix interfacial separations. The proposed XFEM model is verified against the direct finite element method. The model domain, boundary conditions, matrix properties and fiber properties are taken to be the same as in Section 5.1. 


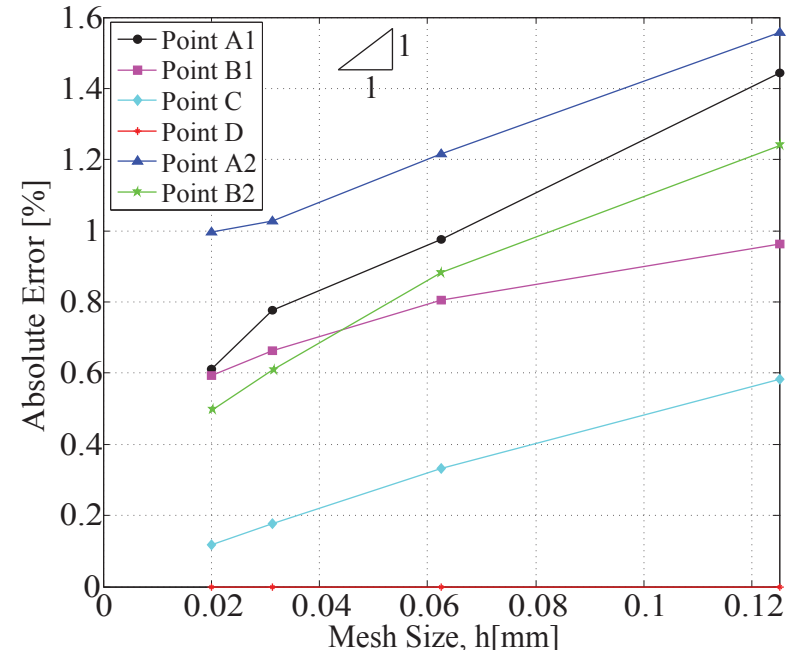

(a)

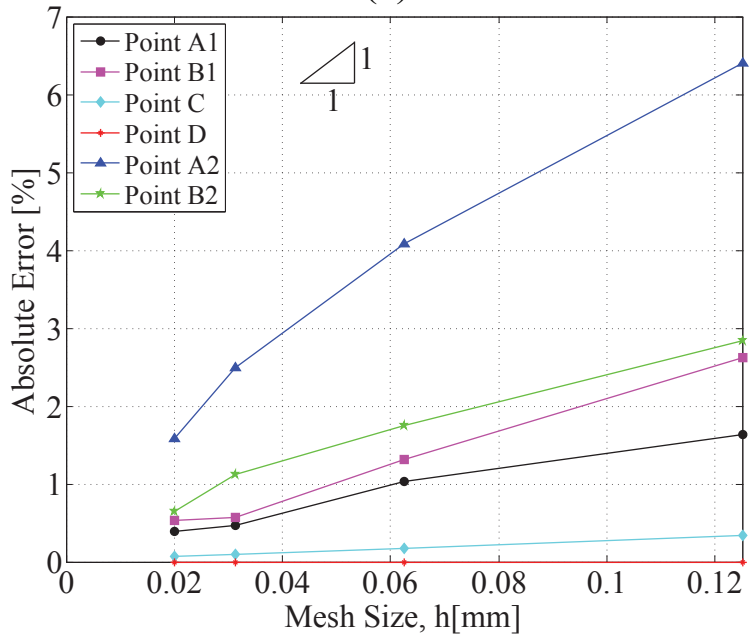

(c)

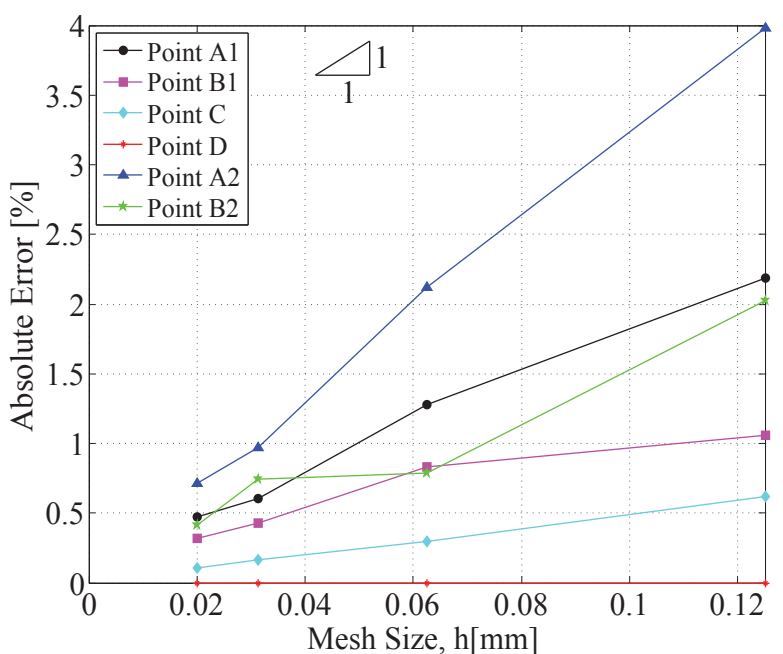

(b)

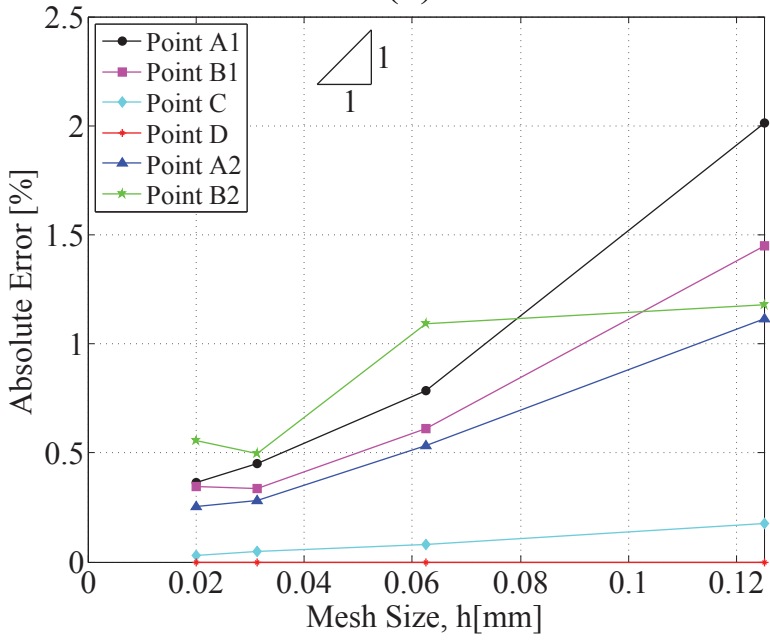

(d)

Figure 7: Point-wise error as function of mesh size for an elastic matrix; (a) case 1; (b) case 2 ; (c) case 3; and (d) case 4. A typical slope of order of magnitude error reduction with order of magnitude decrease of mesh size for the finite element method, is displayed by the legend for comparison. 
To model the progressive debonding at the fiber-matrix interfaces, a bilinear cohesive zone law (defined in Eq. 13 and Eq. 14) is employed for both the XFEM and the reference simulations. The peak normal traction and normal cohesive characteristic separation length are set to $8 \mathrm{MPa}$ and $0.01591 \mathrm{~mm}$, respectively. The peak shear traction and shear cohesive characteristic separation length are $1.8 \mathrm{MPa}$ and $0.01141 \mathrm{~mm}$, respectively. The maximum cohesive separation length is taken as $0.08 \mathrm{~mm}$ under pure normal and pure shear loading $[59,60]$. The reference model utilizes $1 \mu \mathrm{m}$ wide cohesive zone elements that lay along the interface between the fiber and the matrix.

The absolute point-wise displacement error of the proposed model with respect to the reference simulations is shown in Fig. 8. The displacement errors are computed for the same locations of interest in the previous section (points A1, A2, B1, B2, C and D), as well as the displacement jump between the matrix and fiber at each fiber center point.

In Figure 8a, case 1 is shown when the fiber-matrix interfaces have initiated the progressive debonding process but have not reached peak cohesive traction. There is a general trend toward convergence but with some variation at the smallest mesh sizes. The largest error occurred at the right tip of the first fiber, but was approximately $2.5 \%$ at the smallest mesh size. Points C and $\mathrm{D}$ have the lowest error, with both being under $0.1 \%$ for all mesh sizes. The errors of the displacement jumps at the center of both fibers were under $0.5 \%$ for all mesh sizes. Case 2 is shown in Fig. 8b, at the point of full fiber-matrix separation. At the point of full separation, the fiber and the matrix are completely debonded from each other at one side of the fiber and the interfacial tractions vanish. Similar to case 1, there is a trend of towards convergence with a slight variation at the smallest mesh sizes. All errors were at or below $2 \%$. The center jump of fiber 1 produced the highest error at all mesh sizes, but monotonically converged.

The absolute point-wise errors are shown for case 3 and 4 in Fig. 8c and Fig. 8d, respectively. In case 3 , the progressive debonding process has been initiated but has not reached peak cohesive traction. The left fiber tip and center jump of fiber 1 have larger errors than the rest of the points studied, but both have reasonable errors at the smallest mesh size. In case 4, the progressive debonding is past the point of peak cohesive traction but has not fully separated. In this case, there is variation in the monotonic convergence, but the point-wise error remained below $6 \%$ at each point for all mesh sizes, except for the right tip of fiber 1 . The largest errors occurred in the right tips of fiber 1 and fiber 2, which both lie in the same doubly-enriched element. In all four cases studied with multiple fibers, there was not smooth monotonic convergence as observed in the purely elastic models, but general convergence with variation and mesh stability were observed for many of the cases. For all cases, reasonable errors were obtained at the smallest element size.

The magnitude of the displacement jumps associated with the fiber-matrix interfacial debonding along each fiber length for each case is plotted in Figs. 9-13. The proposed XFEM 


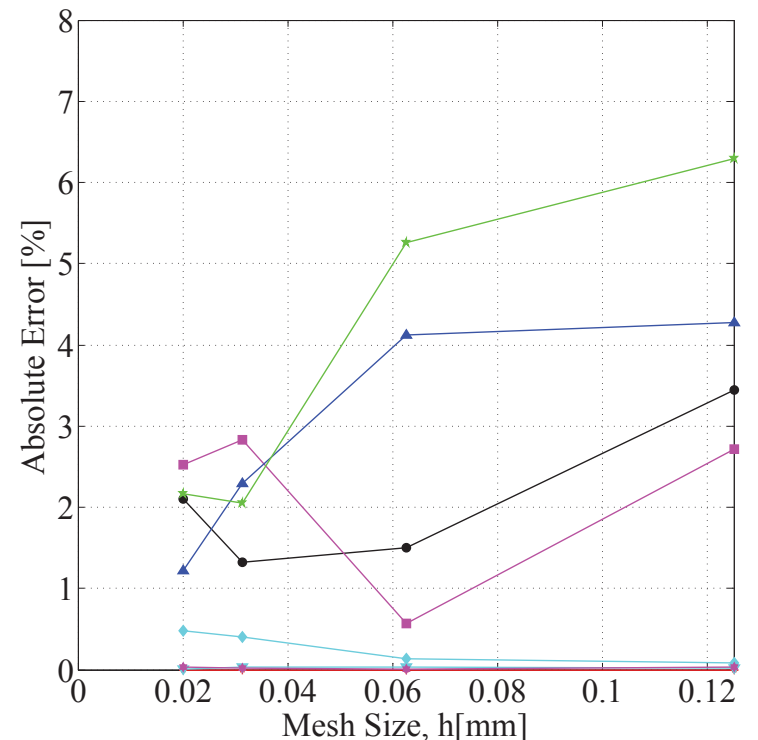

(a)



(c)

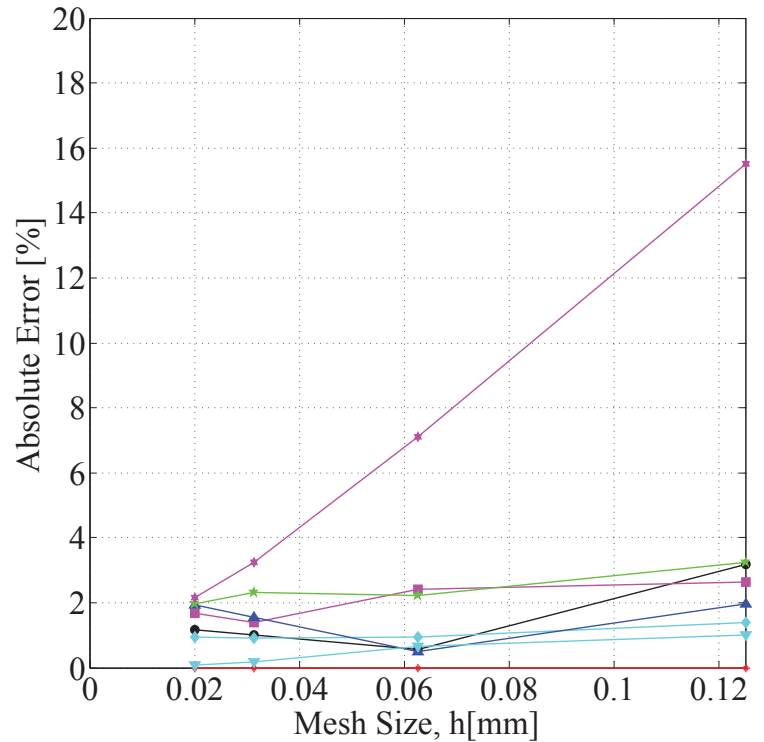

(b)

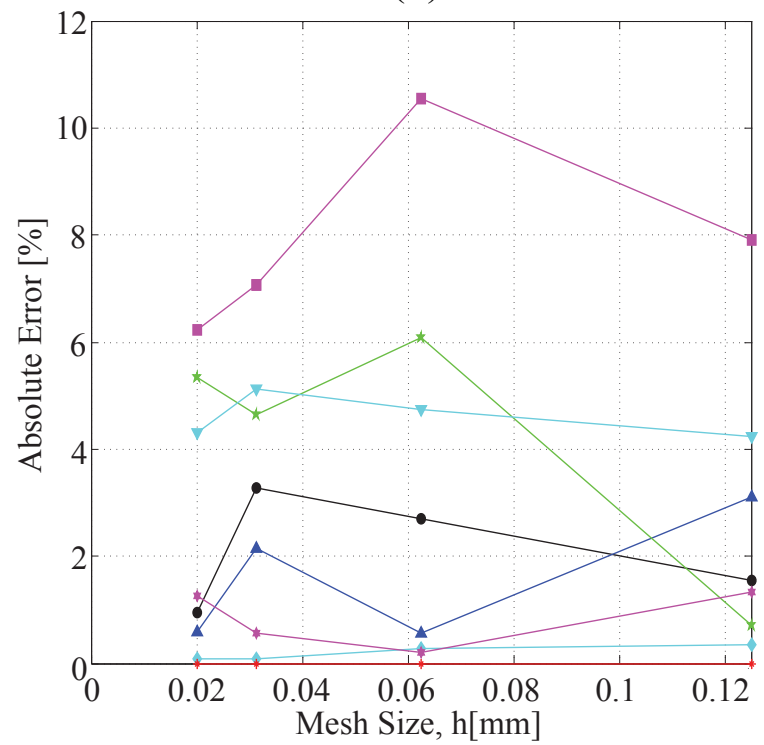

(d)

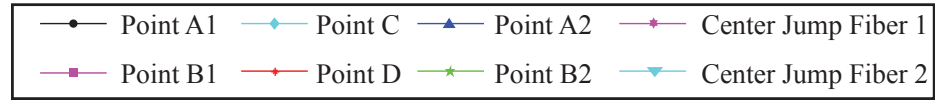

Figure 8: Point-wise error as function of mesh size for a matrix with progressive debonding; (a) case 1 ; (b) case 2; (c) case 3; and (d) case 4. 


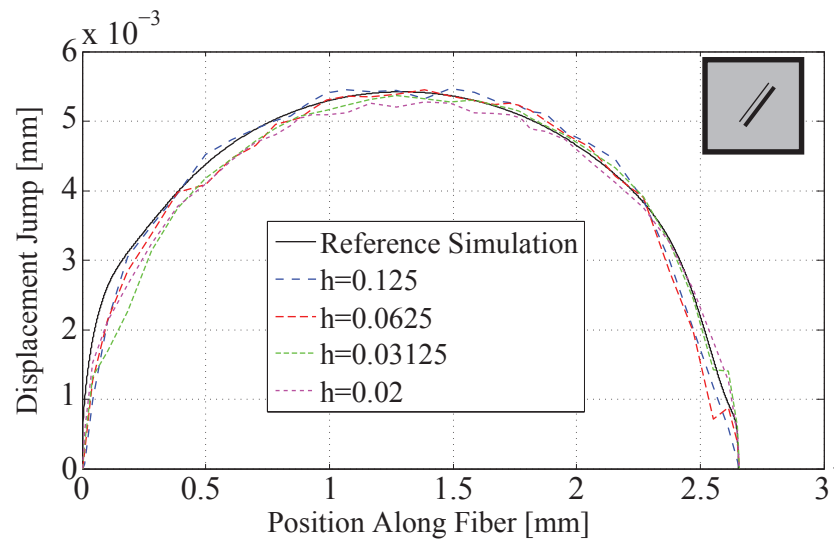

(a)

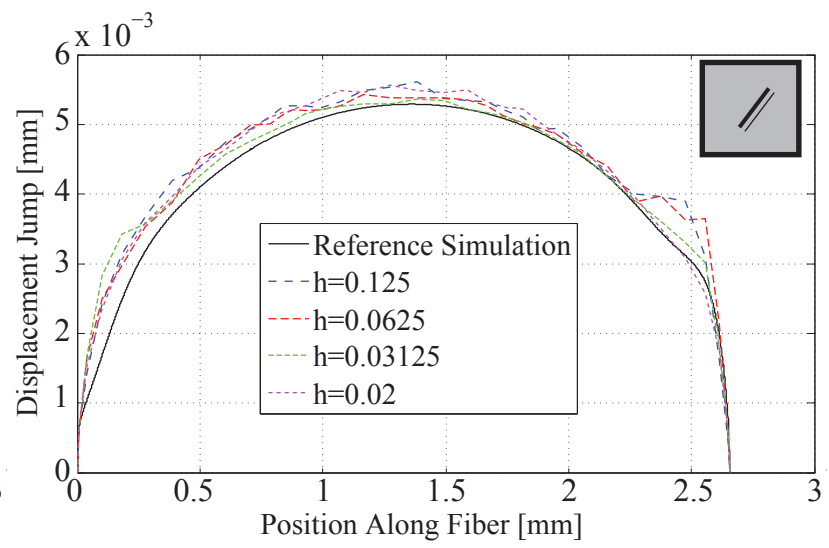

(b)

Figure 9: Displacement jump across the interface along the fiber length ( $h$ is the mesh size) for case 1: (a) fiber 1; and (b) fiber 2 .

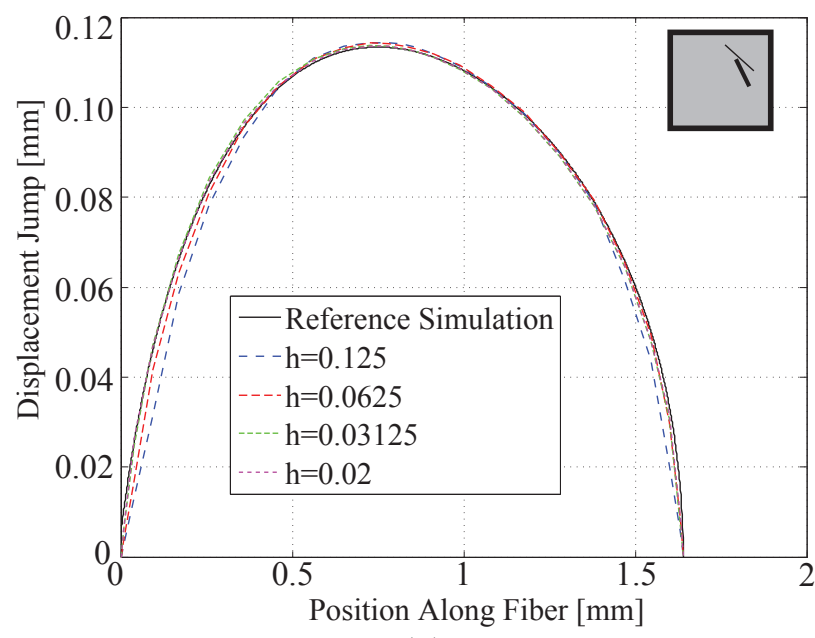

(a)



(b)

Figure 10: Displacement jump across the interface along the fiber length ( $h$ is the mesh size) for case 2: (a) fiber 1; and (b) fiber 2 . 
model results are plotted for the four mesh sizes studied, ranging from $0.125 \mathrm{~mm}$ to $0.02 \mathrm{~mm}$, along with the reference simulation. For each case, the XFEM models accurately capture the shape and magnitude of the reference curve, generally showing improved convergence as the element size decreases. While the general form of the debonding enrichment function is parabolic, asymmetry in the curve as inclusions in close proximity to each other begin to interact is well captured. In all cases, the normal displacement jump is larger than the tangential displacement jump and therefore governs the magnitude of the jump. Fiber tips are assumed to have no debonding in the XFEM and the reference simulations.

In case 1 (Fig. 9), fiber 1 and fiber 2 exhibit approximately the same displacement jump curve in both shape and magnitude. All mesh sizes for case 1 show a reasonably accurate displacement jump profile with mesh stability. A slight kink is observed in the fiber 2 curve near the right end of the fiber, which becomes smoother and converges to the reference simulation as the mesh is refined. The deviation may be attributed to fiber 1 being located between fiber 2 and the applied load. When one fiber "blocks" another fiber from the applied load, the fiber may exhibit an irregular pattern of debonding. This phenomenon is more prominent in case 2 and case 3 , in which each fiber is of different length and has a unique embedment angle. Figure 10 displays the displacement jumps for case 2. Fiber 1, which is above fiber 2, shows a parabolic displacement jump profile, with all four XFEM mesh sizes displaying high accuracy. Fiber 2, which is below fiber 1, shows an irregular displacement jump profile, which is due to having part of fiber 1 directly in front of it. The proposed XFEM model accurately captures the irregular displacement jump profile along the fiber length for all mesh sizes, with the smallest mesh most accurately representing the displacement jump. Figure 11 displays the displacement jumps for case 3 . Similar to case 2, fiber 1 shows a parabolic displacement jump profile and fiber 2 shows an irregular displacement jump profile, which the proposed XFEM model accurately represents. There is a reduction of the maximum displacement jump and convergence with the increase of mesh density observed in this case. Fig. 12 shows the deformed shape of the cohesive zone elements in the respective reference simulation for case 3 , from which the displacement jump profile was obtained (deformation is significantly amplified for visualization purposes). Case 4 is shown in Fig. 13, with both fiber 1 and fiber 2 having roughly parabolic curves, with a slight asymmetry at the right end of each fiber, where the tips meet. Both displacement jump profile curves converged to the shape of the reference simulation as the mesh size was refined.

The lack of monotonic error convergence observed in Fig. 8 merits further discussion. Convergence issues in XFEM can be attributed to a variety of reasons including, but not limited to, the choice of enrichment functions, numerical integration, and partially enriched blending elements [61]. In this study, the lack of monotonic error convergence can be attributed to the limitation of the fiber-matrix debonding enrichment function in representing true debonding 


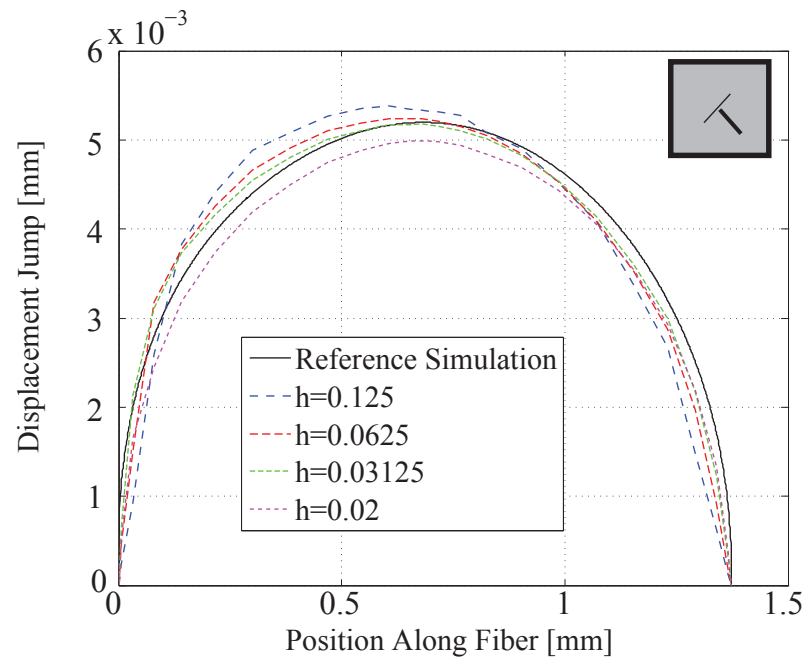

(a)

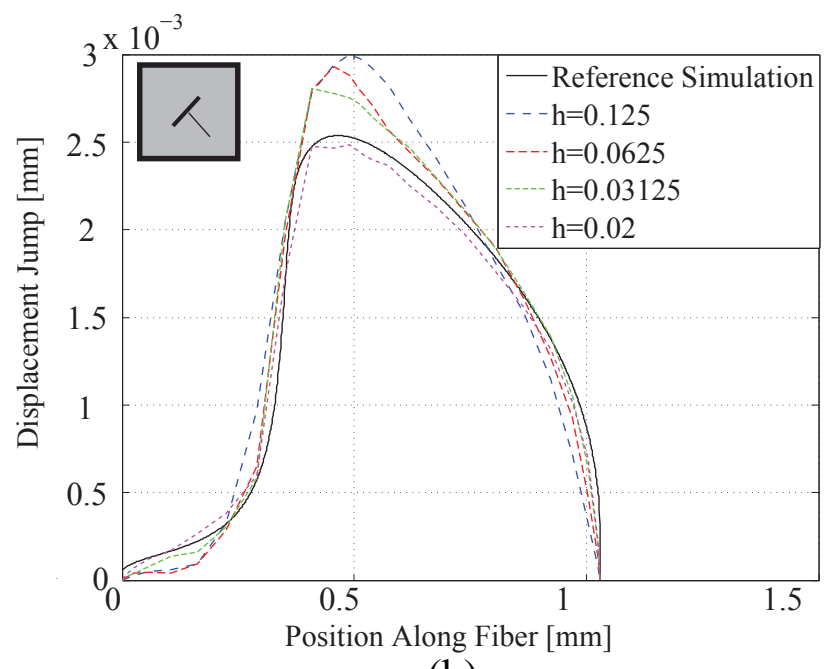

(b)

Figure 11: Displacement jump across the interface along the fiber length ( $h$ is the mesh size) for case 3: (a) fiber 1; and (b) fiber 2 .

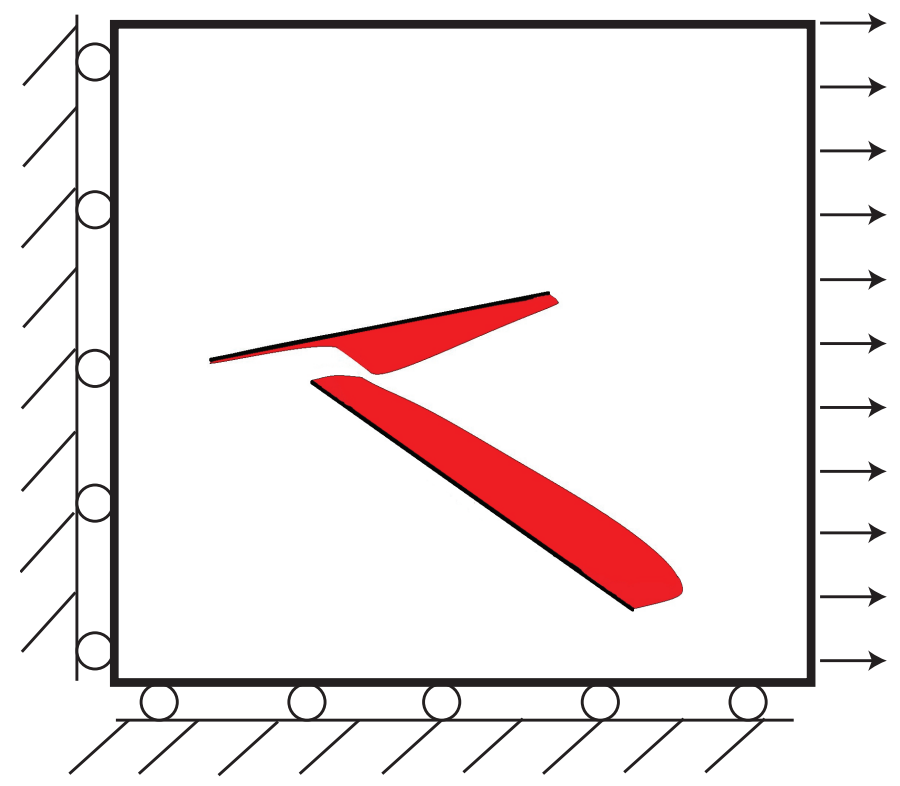

Figure 12: Case 3 reference simulation for progressive debonding between fibers and matrix. Shaded area denotes the displacement jump measured from the cohesive elements, plotted in Fig. 11. Deformation is significantly amplified for visualization purposes. 


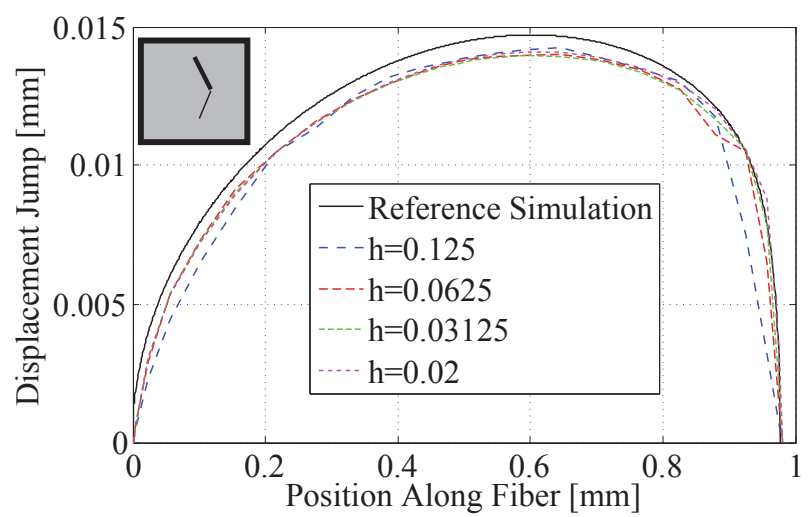

(a)

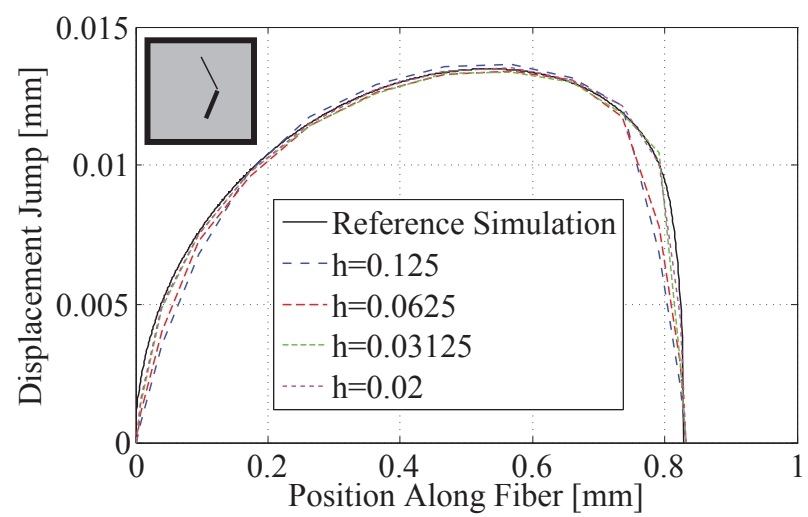

(b)

Figure 13: Displacement jump across the interface along the fiber length ( $h$ is the mesh size) for case 4: (a) fiber 1; and (b) fiber 2 .

profile, as well as the way the enriched element domains (and hence the integration accuracy as discussed above) change as the mesh is refined. In case 1, the right tips of fiber 1 and fiber 2 are in the same element at element lengths of $h=0.125$ and $0.0625 \mathrm{~mm}$, but in different elements at meshes of higher resolution. Thus with a coarse mesh, fiber tip error is computed from a doubly enriched fiber tip element, whereas at fine meshes, the fiber tip error is computed from a tip-enriched element that is partially enriched by the other fiber. In each case, as the mesh is refined, the location of the inclusion with respect to the element edges changes. The spatial alignment of inclusions within an element influences the Delaunay triangulation of the element, which can introduce inconsistencies in the element integration as the size of the element domain changes. 3 Gauss points were used for the integration of each triangular subdomain for the results shown here for the proposed XFEM models. Several simulations were conducted using 6 Gauss points per subdomain but computational cost increased significantly while the measured errors did not improve significantly. Overall, the proposed XFEM model with progressive fibermatrix interfacial debonding provided an accurate representation of the displacement jump curves obtained from the reference simulations and was capable of predicting the displacement jumps for a variety of scenarios in which fibers were arbitrarily placed in close proximity to each other and contained elements with multiple enrichments. Absolute point-wise error was higher than the purely elastic case, but reasonable mesh stability was observed and errors remain within an acceptable range for each case at a sufficiently fine mesh.

Table 1 compares the model size of the reference simulation of progressive debonding of case 1 ( Fig. 6b (i)) with the XFEM simulations for the same case. The number of elements, standard nodes, total nodes and total degree of freedoms (DOFs) associated with each model are displayed. The largest number of elements and DOFs are 62,500 and 130,194, respectively for the finest mesh. The total DOFs of the finest XFEM mesh are smaller by approximately 


\begin{tabular}{|c|c|c|c|c|c|}
\hline & & \multicolumn{4}{|c|}{ XFEM } \\
& Reference Model & $\mathrm{h}=0.125$ & $\mathrm{~h}=0.0625$ & $\mathrm{~h}=0.03125$ & $\mathrm{~h}=0.02$ \\
\hline Number of Elements & 601,236 & 400 & 6,400 & 25,600 & 62,500 \\
\hline Number of Standard Nodes & 601,427 & 1,681 & 6,561 & 25,921 & 63,001 \\
\hline Number of Total Nodes & 601,427 & 2,051 & 7,255 & 27,269 & 65,097 \\
\hline Number of Total DOFs & $1,202,854$ & 4,102 & 14,510 & 54,538 & 130,194 \\
\hline
\end{tabular}

Table 1: Problem size comparison for debonding case 1.



Figure 14: Fiber configuration of four fiber inclusions interaction case.

an order of magnitude compared to the reference simulation.

\subsection{Progressive debonding interactions of four fiber inclusions}

We investigate the performance of the proposed XFEM model for the progressive debonding response for a four fiber inclusion case when the inclusions are in close proximity of each other, but not touch or overlap. The fibers are placed, such that various elements in the domain may contain up to three fiber inclusions, for all mesh sizes studied. The model domain, boundary conditions, matrix properties and fiber properties are taken to be the same as in Section 5.1. The cohesive law used for the progressive debonding at the fiber-matrix interfaces is the same used in Section 5.2.

The case consisting of four fiber inclusions, contains fibers which are in close proximity to each other as illustrated in Fig. 14. Fiber 1 is located in the center of the domain between three other fibers, with a length of $2.34 \mathrm{~mm}$ and an angle of 38 degrees measured counterclockwise from the horizontal. Fiber 2 is measured at a length of $0.9 \mathrm{~mm}$ and an angle of -54 degrees. Fiber 3 is placed so that the right tip of fiber 3 lies in the same element at the left tip of fiber 2 and the domain of fiber 1 . The lengths and angles of fibers 3 and 4 are 1.25mm, -27 degrees and $1.62 \mathrm{~mm}, 58$ degrees, respectively.

The fiber-matrix interfacial progressive debonding displacement jumps are illustrated in 


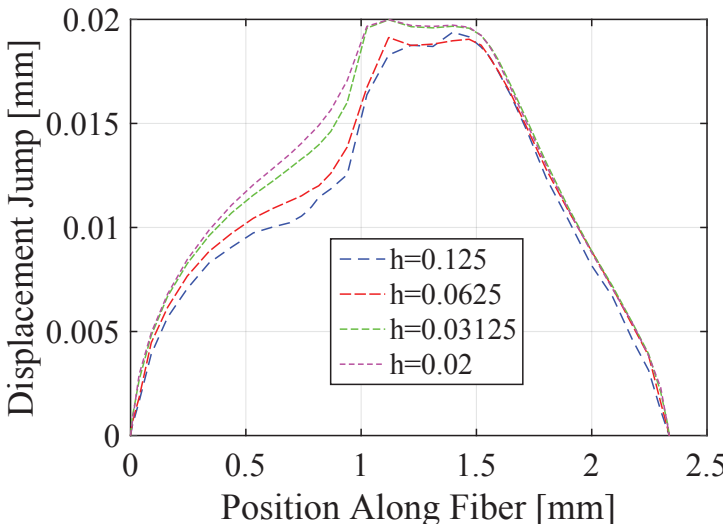

(a)

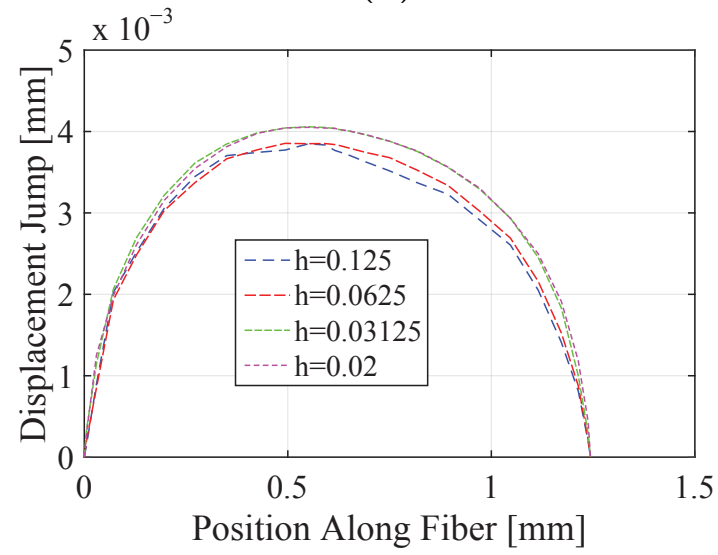

(c)

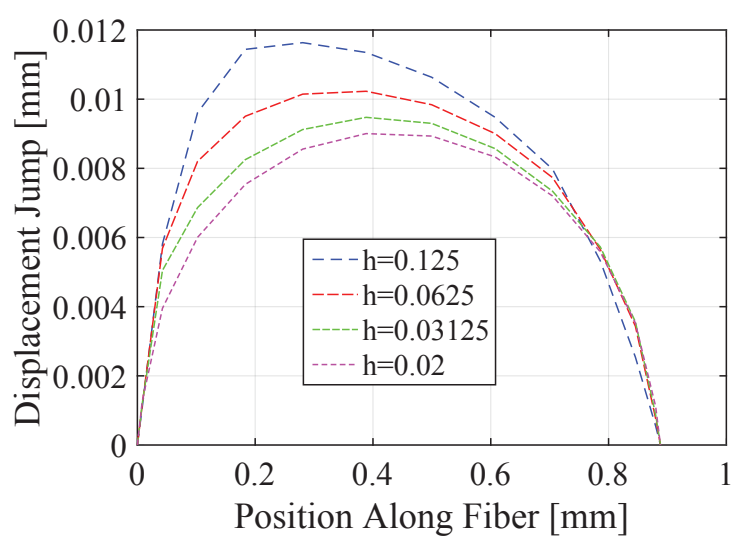

(b)

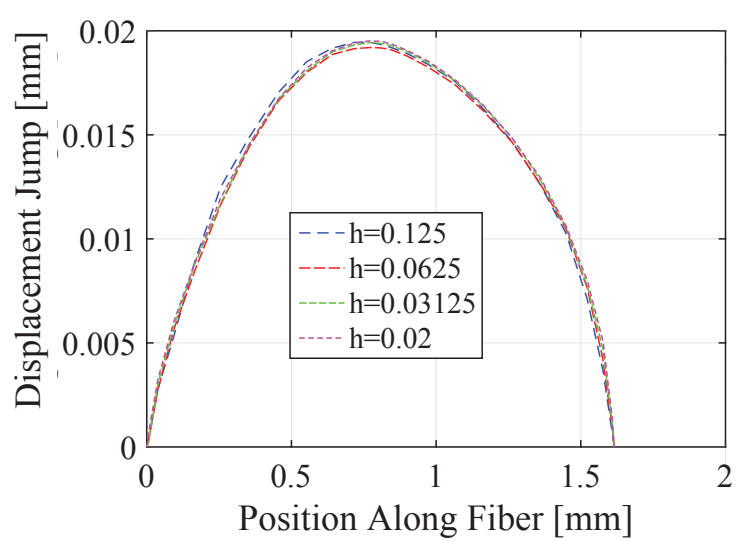

(d)

Figure 15: Displacement jump across the interface along the fiber length ( $h$ is the mesh size) for the four fiber configuration case: (a) fiber 1; (b) fiber 2; (c) fiber 3; and (d) fiber 4. 
Fig. 15. The interaction responses between the fibers are captured with the displacement jump of each fiber, along the fiber length. In this case, we only consider the proposed XFEM model for mesh sizes ranging from $0.125 \mathrm{~mm}$ to $0.02 \mathrm{~mm}$. For fiber 1 and fiber 4 , the progressive debonding is past the point of peak cohesive traction but has not fully separated. In fiber 2 and fiber 3, the progressive debonding process has been initiated but has not reached peak cohesive traction. Figure 15 displays the shape and magnitude of the displacement jumps for each of the four fibers, showing convergence as the element size decreases. Each of the fiber displacement jumps do not exhibit a perfect parabola, but show an asymmetric displacement jump profile due to the interactions from the close proximity of each of the fibers.

The displacement jump profile for fiber 1, illustrated in Fig. 15a, exhibits an irregular jump profile. The irregular displacement jump profile is due to the having part of fiber 2 in front of it, as well as the tips from fibers 3 and 4 on the left side of the fiber domain. The peak displacement jump occurs at the midpoint of the fiber. Figure 15b displays an asymmetric displacement jump profile jump of fiber 2, with the peak occurring left of the fiber midpoint. Fibers 3 and 4 display a parabolic shaped displacement jump profile represented in Fig. 15c and Fig. 15d, respectively. Each of the fiber displacement jump profiles exhibited mesh convergence with the decrease in mesh size.

\subsection{Progressive debonding of a dense fiber domain}

In this section, investigation of a dense fiber domain is performed with the proposed XFEM model for the progressive debonding response. 50 fibers are randomly placed in a $10 \mathrm{~mm}$ by $10 \mathrm{~mm}$ domain with the fibers having a mean length of $1.3 \mathrm{~mm}( \pm 0.2 \mathrm{~mm})$, as displayed in Fig. 16. With the random dispersion of fibers, multiple elements have multiple fiber enrichments in them. The mesh size is $\mathrm{h}=1 \mathrm{~mm}$ in this example. The boundary conditions, matrix properties and fiber properties are taken to be the same as in Section 5.1. The cohesive law used for the progressive debonding at the fiber-matrix interfaces is the same used in Section 5.2.

The displacement jumps between the fiber and the matrix for each of the fibers are summarized in Fig. 17. Fig. 17a displays the number of fibers for their respective maximum magnitude of separation of the displacement jumps. 2 fibers resulted in a maximum interface separation of less than $0.01 \mathrm{~mm}$ while there were 19 fibers for a maximum separation between 0.01-0.25 $\mathrm{mm}$ as well as 0.025-.05 mm. 9 fibers showed maximum separation between 0.05-.075 mm, and a single fiber showed an interface separation greater than $0.075 \mathrm{~mm}$ with complete loss of interfacial cohesion. For the 50 total fibers in the domain, 5 did not separate (i.e. maximum separation less than the characteristic separation length), 44 fiber matrix interfaces were partially debonded and only 1 fiber matrix interface had a maximum separation that resulted in complete separation. Fig. 17b displays the severity of debonding. 


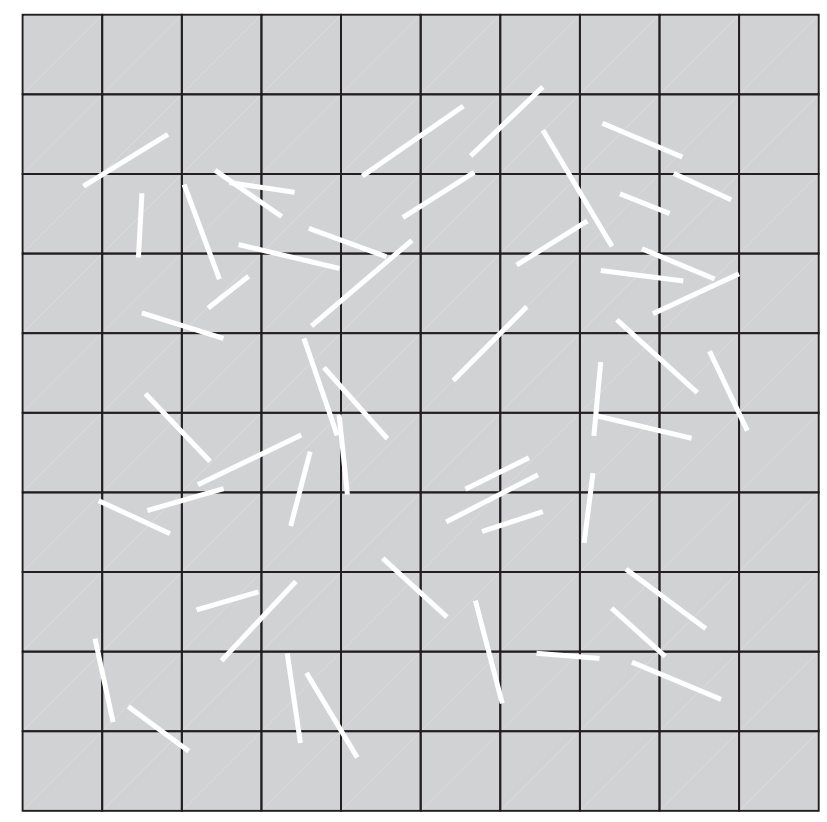

Figure 16: Dense fiber domain case.

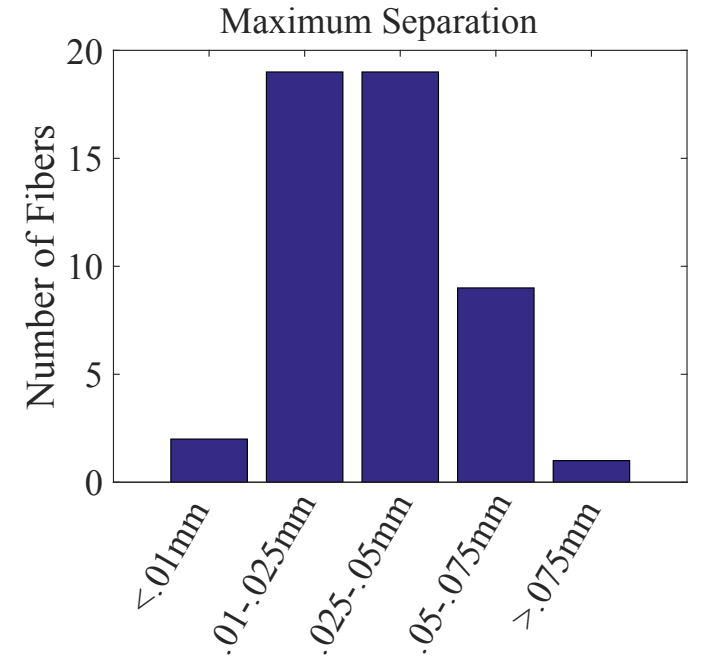

(a)



Figure 17: Summary of displacement jumps for the fibers in the dense fiber domain: (a) Total number of fibers with their respective maximum separation of each displacement jump; (b) Total number of fibers with no separation, partial separation and full separation. 


\section{Conclusions}

This manuscript presented the formulation and implementation of an XFEM model to capture the interactions between fibers in short fiber reinforced composites. The fiber inclusions were modeled as elastic objects of zero measure. Inclusion and debonding enrichment functions were included to model the fibers and the progressive fiber-matrix debonding within the XFEM framework. This approach eliminates the need to use finite element meshes compliant with fiber inclusions and cohesive zone models which increase computational cost. Numerical integration procedures were provided for accurate evaluation of the system response for randomly positioned fibers, including for multiple fibers that occupy the same element. The performance of the XFEM model was assessed against the direct finite element method for various multiple fiber domain configurations in two dimensions. The proposed approach accurately captures the elastic and progressive debonding interaction behavior between multiple short fibers in reinforced composites without the need for mesh compliance. The ability to include multiple enrichments in the same element provides the capability to increase fiber density in the composite microstructure without significantly increasing the base finite element grid density, allowing the capability to simulate realistic microstructures with a large number of fibers. The present approach is significantly more computational efficient compared to a finite element analysis, in which the microstructure is fully resolved as demonstrated in the numerical examples. The computational efficiency of the present approach remains too high to be included in a concurrently coupled multiscale simulation, yet presents an efficient approach for sequential multiscale algorithms, as well as investigations of failure mechanisms within composite microstructures.

Important advancements to the proposed model are under development. The debonding enrichment function proposed in this manuscript assumes no debonding at the fibers tips. In reality, this is not the case and fiber end slip will be considered in future work to account for this deformation. The distribution of the fibers can also be studied to understand the effect of placement within the domain. This manuscript provided the formulation and implementation

details for two-dimensional problems only. The proposed formulation is being extended to three dimensions, which provides many significant challenges particularly in the computational implementation as well as modeling the progressive debonding between fibers modeled as objects of zero measure in the three dimensional matrix. Our near term research efforts will focus on extending the proposed modeling approach to three dimensions. 


\section{Acknowledgments}

The authors gratefully acknowledge the financial support from Vanderbilt University through the Discovery grant program and the Vanderbilt Institute of Software Integrated Systems.

\section{References}

[1] J. Won, B. Hong, T. Choi, S. Lee, and J. Kang. Flexural behaviour of amorphous microsteel fibre-reinforced cement composites. Comp. Struct., 94:1443-1449, 2012.

[2] D. Yoo, J. Lee, and Y. Yoon. Effect of fiber content on mechanical and fracture properties of ultra high performance fiber reinforced cementitious composites. Comp. Struct., 106: 742-753, 2013.

[3] V.C. Li. On engineered cementitious composites (ECC) - a review of the material and its applications. J. of Advanced Concrete Technol., 1:215-230, 2003.

[4] D.D.L. Chung. Cement reinforced with short carbon fibers: a multifunctional material. Composites Part B: Engineering, 31:511-526, 2000.

[5] D.D.L. Chung. Composites get smart. Materials Today, 5:30-35, 2002.

[6] F. Reza, J. A. Yamamuro, and G. B. Batson. Electrical resistance change in compact tension specimens of carbon fiber cement composites. Cement and Concrete Compos., 26: 873-881, 2004.

[7] X. Fu and D. D. L. Chung. Submicron carbon filament cement-matrix composites for electromagnetic interference shielding. Cement and Concrete Research, 26:1467-1472, 1996.

[8] G. P. Tandon and G. J. Weng. Average stress in the matrix and effective moduli of randomly oriented composites. Compos. Sci. Technol., 27:111-132, 1986.

[9] J. H. Huang. Some closed-form solutions for effective moduli of composites containing randomly oriented short fibers. Mater. Sci. Engng. A, 315:11-20, 2001.

[10] A. Bouaziz, M. Nait-Abdelaziz, J. M. Gloaguen, and J. M. Lefebvre. Micromechanical modelling and experimental investigation of random discontinuous glass fiber polymermatrix composites. Compos. Sci. Technol., 67:3278-3285, 2007.

[11] P. Ponte-Castaneda and J. R. Willis. The effect of spatial distribution on the effective behavior of composite materials and cracked media. J. Mech. Phys. Solids, 43:1919-1951, 1995. 
[12] H. J. Bohm, A. Eckschlager, and W. Han. Multi-inclusion unit cell models for metal matrix composites with randomly oriented discontinuous reinforcements. Comp. Mater. Sci., 25:42-53, 2002.

[13] H. R. Lusti, P. J. Hine, and A. A. Gusev. Direct numerical predictions for the elastic and thermoelastic properties of short fibre composites. Compos. Sci. Technol., 62:1927-1934, 2002 .

[14] R. D. Crouch, S. B. Clay, and C. Oskay. Experimental and computational investigation of progressive damage accumulation in cfrp composites. Compos. Part B: Engng., 48: 59.-67, 2013.

[15] A.G. Evans and F.W. Zok. The physics and mechanics of fibre-reinforced brittle matrix composites. J. of Mat. Sci., 29:3857-3896, 1994.

[16] L. Mishnaevsky Jr. and P. Brndsted. Three-dimensional numerical modelling of damage initiation in unidirectional fiber-reinforced composites with ductile matrix. Mater. Sci. and Engng. A, 498:81-86, 2008.

[17] J.L. Winds, S. Steffensen, and H.M. Jensen. Comparison of a composite model and an individually fiber and matrix discretized model for kink band formation. Int. J. Non-Lin. Mech., 67:319-325, 2014.

[18] D. Coronelli and M.G. Mulas. Local global approach in the seismic analysis of R/C frames including bond slip effects. Engng. Struct., 23:911-925, 2001.

[19] T. Rabczuk, J. Akkermann, and J. Eibl. A numerical model for reinforced concrete structures. Int. J. Solids and Struct., 42:1327-1354, 2005.

[20] N. Moës, J. Dolbow, and T. Belytschko. A finite element method for crack growth without remeshing. Int. J. Numer. Meth. Engng., 46:131-150, 1999.

[21] T. Belytschko and T. Black. Elastic crack growth in finite elements with mimal remeshing. Int. J. Numer. Meth. Engng., 45:601-620, 1999.

[22] C. A. Duarte, I. Babuska., and J. Oden. Generalized finite element methods for threedimensional structural mechanics problems. Computers and Structures, 77:215-232, 2000.

[23] I. Babuska and J. M. Melenk. The partition of unity method. Int. J. Numer. Meth. Engng., 40:727-758, 1997.

[24] N. Sukumar, N. Moes, B. Moran, and T. Belytschko. Extended finite element method for three-dimensional crack modelling. Int. J. Numer. Meth. Engng., 48:1549-1570, 2000. 
[25] T. Belytschko, N. Moes, S. Usui, and C. Parimi. Arbitrary discontinuities in finite elements. Int. J. Numer. Meth. Engng., 50:993-1013, 2001.

[26] N. Sukumar and J-H. Prevost. Modeling quasi-static crack growth with the extended finite element method part 1: Computer implementation. Int. J. Solids and Struct., 40: 7513-7537, 2003.

[27] T. Fries and T. Belytschko. The intrinsic XFEM: a method for arbitrary discontinuities without additional unknowns. Int. J. Numer. Meth. Engng., 68:1358-1385, 2006.

[28] M. Lan, H. Waisman, and I. Harari. A high-order extended finite element method for extraction of mixed-mode strain energy release rates in arbitrary crack settings based on Irwins integral. Int. J. Numer. Meth. Engng., 96:787-812, 2013.

[29] J. Pan and C. K. Y. Leung. Debonding along the FRP - concrete interface under combined pulling/peeling effects. Engng. Fract. Mech., 74:132-150, 2007.

[30] Y. T. Obaidat, S. Heyden, and O.Dahlblom. The effect of CFRP and CFRP/concrete interface models when modelling retrofitted RC beams with FEM. Comp. Struct., 92: 1391-1398, 2010.

[31] I.G. Garcia, M. Paggi, and V. Mantic. Fiber-size effects on the onset of fiber-matrix debonding under transverse tension: A comparison between cohesive zone and finite fracture mechanics models. Engng. Fract. Mech., 115:96-110, 2014.

[32] N. Moës and T. Belytschko. Extended finite element method for cohesive crack growth. Engng. Fract. Mech., 69:813-833, 2002.

[33] J. Unger, S. Eckardt, and C. Konke. Modelling of cohesive crack growth in concrete structures with the extended finite element method. Comput. Methods Appl. Mech. Engng., 196:4087-4100, 2007.

[34] G. Zi and T. Belytschko. New crack-tip elements for XFEM and applications to cohesive cracks. Int. J. Numer. Meth. Engng., 57:2221-2240, 2003.

[35] J.L. Asferg, P.N. Poulsen, and L.O. Nielsen. A consistent partly cracked XFEM element for cohesive crack growth. Int. J. Numer. Meth. Engng., 72:464-485, 2007.

[36] T.P. Fries, A. Byfut, A. Alizada, K. Chen, and A. Schroder. Hanging nodes and xfem. Int. J. Numer. Meth. Engng., 86:404-430, 2011.

[37] E. Budyn, G. Zi, N. Moes, and T. Belytschko. A method for multiple crack growth in brittle materials without remeshing. Int. J. Numer. Meth. Engng., 61:1741-1770, 2004. 
[38] C. Richardson, J. Hegemann, E. Sifakis, J. Hellrung, and J. Teran. An XFEM method for modeling geometrically elaborate crack propagation in brittle materials. Int. J. Numer. Meth. Engng., 88:1042-1065, 2011.

[39] C Daux, N. Moes, J. Doblow, N. Sukumar, and T.Belytschko. Arbitrary branched and intersecting cracks with the extended finite element method. Int. J. Numer. Meth. Engng., 48:1741-1760, 2000.

[40] G. Zi, J. Song, E. Budyn, S. Lee., and T. Belytschko. A method for growing multiple cracks without remeshing and its application to fatigue crack growth. Modelling Simul. Mater. Sci. Engng., 12:901-915, 2004.

[41] B. Hiriyur, H. Waisman, and G. Deodatis. Uncertainty quantification in homogenization of heterogeneous microstructures modeled by XFEM. Int. J. Numer. Meth. Engng., 88: $257-278,2011$.

[42] A.E. Elwi and T.M Hrudey. Finite element model for curved embedded reinforcement. J. Engng. Mech., 115:740-754, 1989.

[43] F. Barzegar and S. Maddipudi. Three dimensional modelling of conrete structures. ii: Reinforced concrete. J. Struct. Engng., 123:1347-1356, 1997.

[44] H. Hartl. Development of a continuum mechanics based tool for 3D FEA of RC Structures and application to problems of soil structure interaction. $\mathrm{PhD}$ thesis, Graz Univ. of Technology, 2002.

[45] G.C. Lykidis and K.V. Spiliopoulus. 3d solid finite element analysis of cylically loaded RC structures allowing embedded reinforcment slippage. J. Struct. Engng., 134:629-638, 2008.

[46] F.K.F. Radtke, A. Simone, and L.J. Sluys. A partition of unity finite element method for obtaining elastic properties of continua with embedded thin fibres. Int. J. Numer. Meth. Engng., 84:708-732, 2010.

[47] F.K.F. Radtke, A. Simone, and L.J. Sluys. A partition of unity finite element method for simulating non-linear debonding and matrix failure in thin fibre composites. Int. J. Numer. Meth. Engng., 86:453-476, 2011.

[48] J.C. Simo, J. Oliver, and F. Armero. An analysis of strong discontinuities induced by strain-softening in rate-independent inelastic solids. Comp. Mech., 12:277-296, 1993. 
[49] M. Jirasek. Comparative study on finite elements with embedded discontinuities. Comput. Meth. Appl. Mech. Engng., 188:307-330, 2000.

[50] A. Hansbo and P. Hansbo. A finite element method for the simulation of strong and weak discontinuities in solid mechanics. Comput. Meth. Appl. Mech. Engng., 193:3523-3540, 2004.

[51] J. Fish and A. Wagiman. Multiscale finite element method for heterogeneous medium. Comp. Mech.: Int. J., 12:1-17, 1993.

[52] J. Fish. Hierarchical modeling of discontinuous fields. Comm. Appl. Num. Meth., 8: 443-453, 1992.

[53] M. Pike and C. Oskay. Modeling random short nanofiber- and microfiber-reinforced composites using the extended finite-element method. J. Nanomech. Micromech., 5:1-11, 2015.

[54] M. Pike and C. Oskay. XFEM modeling of short microfiber reinforced composites with cohesive interfaces. Finite Elements in Analysis and Design, 106:16-31, 2015.

[55] F. Sanchez and C. Ince. Microstructure and macroscopic properties of hybrid carbon nanofiber/silica fume cement composites. Compos. Sci. Technol., 69:1310-1318, 2009.

[56] N. Moës, M. Cloirec, P. Cartraud, and J.-F. Remacle. A computational approach to handle complex microstructure geometries. Comput. Methods Appl. Mech. Engng., 192: 3163-3177, 2003.

[57] L. Chen, T. Rabczuk, S. P. A. Bordas, G. R. Liu, K. Y. Zeng, and P. Kerfriden. Extended finite element method with edge-based strain smoothing (ESm-XFEM) for linear elastic crack growth. Comput. Methods Appl. Mech. Engng., 209:250-265, 2012.

[58] T.P. Fries. A corrected XFEM approximation without problems in blending elements. Int. J. Numer. Meth. Engng., 75:503-532, 2008.

[59] X.-P. Xu and A. Needleman. Numerical simulations of fast crack growth in brittle solids. J. Mech. Phys. Solids, 42:1397-1434, 1994.

[60] J. Wang. Cohesive zone model of intermediate crack-induced debonding of FRP-plated concrete beam. Int. J. Solids and Struct., 43:6630-6648, 2006.

[61] Gordeliy E. and A. Peirce. Enrichment strategies and convergence properties of the XFEM for hydraulic fracture problems. Comput. Meth. Appl. Mech. Engng., 283:474-502, 2015. 